CERN-TH/99-38

SLAC-PUB-8068

SU-ITP-98/68

\title{
Rapid Asymmetric Inflation and Early Cosmology in Theories with Sub-Millimeter Dimensions
}

\author{
Nima Arkani-Hamed ${ }^{a}$, Savas Dimopoulos ${ }^{b}$, Nemanja Kaloper $^{b}$, \\ and John March-Russell ${ }^{c}$ \\ ${ }^{a}$ SLAC, Stanford University, Stanford, CA 94309, USA \\ ${ }^{b}$ Physics Department, Stanford University, Stanford CA 94305, USA \\ ${ }^{c}$ Theory Division, CERN, CH-1211, Geneva 23, Switzerland
}

\begin{abstract}
It was recently pointed out that the fundamental Planck mass could be close to the $\mathrm{TeV}$ scale with the observed weakness of gravity at long distances being due the existence of new sub-millimeter spatial dimensions. In this picture the standard model fields are localized to a $(3+1)$-dimensional wall or "3-brane". We show that in such theories there exist attractive models of inflation that occur while the size of the new dimensions are still small. We show that it is easy to produce the required number of efoldings, and further that the density perturbations $\delta \rho / \rho$ as measured by COBE can be easily reproduced, both in overall magnitude and in their approximately scaleinvariant spectrum. In the minimal approach, the inflaton field is just the moduli describing the size of the internal dimensions, the role of the inflationary potential being played by the stabilizing potential of the internal space. We show that under quite general conditions, the inflationary era is followed by an epoch of contraction of our world on the brane, while the internal dimensions slowly expand to their stabilization radius. We find a set of exact solutions which describe this behavior, generalizing the well-known Kasner solutions. During this phase, the production of bulk gravitons remains suppressed. The period of contraction is terminated by the blue-shifting of Hawking radiation left on our wall at the end of the inflationary de Sitter phase. The temperature to which this is reheated is consistent with the normalcy bounds. We give a precise definition of the radion moduli problem.
\end{abstract}




\section{Introduction}

It was recently pointed out that the fundamental Planck mass could be close to the TeV scale [1, 2, 3, 4], thus providing a novel solution to the hierarchy problem for the standard model. Gravity becomes comparable in strength to the other interactions at this scale, and the observed weakness of gravity at long distances is then explained by the presence of $n$ new "large" spatial dimensions. Gauss' Law relates the Planck scales of the $(4+n)$ dimensional theory, $M_{*}$, and the long-distance 4-dimensional theory, $M_{\mathrm{pl}}$,

$$
M_{\mathrm{pl}}^{2}=\left(b_{0}\right)^{n} M_{*}^{n+2}
$$

where $b_{0}$ is the (present, stabilized) size of the extra dimensions. If we put $M_{*} \sim 1 \mathrm{TeV}$ then

$$
b_{0} \sim 10^{-17+\frac{30}{n}} \mathrm{~cm}
$$

For $n=1, b_{0} \sim 10^{13} \mathrm{~cm}$, so this case is excluded since it would modify Newtonian gravitation at solar-system distances. Already for $n=2$, however, $b_{0} \sim 1 \mathrm{~mm}$, which happens to be the distance where our present experimental knowledge of gravitational strength forces ends. For larger $n, 1 / b_{0}$ slowly approaches the fundamental Planck scale $M_{*}$.

While the gravitational force has not been measured beneath a millimeter, the success of the SM up to $\sim 100 \mathrm{GeV}$ implies that the SM fields can not feel these extra large dimensions; that is, they must be stuck on a 3-dimensional wall, or "3-brane", in the higher dimensional space. Thus, in this framework the universe is $(4+n)$-dimensional with fundamental Planck scale near the weak scale, with $n \geq 2$ new sub-mm sized dimensions where gravity, and perhaps other fields, can freely propagate, but where the SM particles are localized on a 3-brane in the higher-dimensional space. The most attractive possibility for localizing the SM fields to the brane is to employ the D-branes that naturally occur in type I or type II string theory [5, 2]. Gauge and other degrees of freedom are naturally confined to such D-branes [5], and furthermore this approach has the obvious advantage of being formulated within a consistent theory of gravity. However, from a practical point of view, the most important question is whether this framework is experimentally excluded. This was the subject of [3] where laboratory, astrophysical, and cosmological constraints were studied and found not to exclude these ideas.

There are a number of other papers discussing related suggestions. Refs. [6] examine the idea of lowering the gauge-coupling unification scale by utilizing higher dimensions. Further papers concern themselves with the construction of string models with 
extra dimensions larger than the string scale [7, 8, 9], gauge coupling unification in higher dimensions without lowering the unification scale [10], the effective theory of the low energy degrees of freedom in realizations of our world as a brane [11] and radius stabilization [12, 4]. There have also been many recent papers discussing various theoretical and phenomenological aspects of this scheme [13], and a few papers on aspects of the early universe cosmology [14] [15] [16] [17] [18] that discuss issues related to those considered here. f $^{-1}$

In this paper we will discuss inflation and other general aspects of early universe cosmology in world-as-a-brane models. In particular we will be concerned with aspects of early universe cosmology that involve the dynamics of the internal dimensions in a central way. We find that there exist attractive models of inflation that occur while the internal dimensions are still small, far away from their final stabilized value given by the Gauss' law constraint. We show that in such models it is very easy to produce the required number of efoldings of inflation even though no energy densities exceed the fundamental Planck scale, $\rho<M_{*}^{4}$, and that supersymmetry, if it exists at all, is very badly broken on our brane. Further, we demonstrate that the density perturbations $\delta \rho / \rho$ as measured by COBE and the other microwave background and large-scale structure experiments can be easily reproduced in such models, both in their magnitude and in their approximately scale-invariant spectrum. In the most minimal approach, the inflaton field may be just the moduli describing the size of the internal dimensions itself (the radion field of 四), the role of the inflationary potential being played by the stabilizing potential of the internal space. In the case of a wall-localized inflaton, the cosmological constant might even result from the electroweak phase transition, in which case the inflaton is the Higgs. Actually, an important remark in this regard is that when the internal dimensions are small, $b \sim M_{*}^{-1}$, the distinction between on-thewall and off-the-wall physics is not meaningful: e.g., the inflationary features in $V(b)$ at small $b$ could be due to Higgs physics on the wall.

The approximately scale-invariant nature of the primordial perturbation spectrum

* However, we note that a closer scrutiny of 18 reveals that it is impossible to get any inflation at all in the models considered. The potentials in 18 all have exponential dependence on the radion field, and thus lead to the power-law dependence of the scale factor on time, $\bar{a} \sim t^{2 / \beta^{2}}$ [21]. The correct formula for the parameter $\beta$ of $[18$ is $\beta=2 \sqrt{2 n /(n+2)}$, and thus $\beta \geq 2$ for all cases $n \geq 2$, which gives only subluminal expansion, and not inflation. In fact, a complete analysis must employ the physically meaningful measure of scales, namely the scale factor expressed in units of the Compton wavelength of wall particles. (Because of the possibility of Weyl rescaling between string and Einstein frames one must be careful about the definition of physical quantities.) In such physical units it is possible to show that the scale factor always expands sub-luminally, even more slowly than the naive Einstein frame expansion, and thus none of the solutions of [18] contain a stage of inflation. 
implies that, during inflation, the internal dimensions must expand more slowly than the universe on the wall. Thus we are led to consider a form of asymmetric inflationary expansion of the higher-dimensional world. It is very interesting to note that requiring the consistency and naturalness of both the long duration of inflation (i.e., number of efolds $N_{e} \gtrsim 100$ ), and the magnitude of $\delta \rho / \rho$, implies that inflation should occur early, with the internal dimensions close to their natural initial size $b_{i} \sim M_{*}^{-1}$. In particular, we avoid the introduction of extremely light inflatons which seem to be needed if inflation occurs after the internal dimensions reach their current, large, size [14, 15]. We emphasize here that this constraint emerged from the assumption that during inflation the internal space was already large and stable. In fact, in such scenarios obtaining $\delta \rho / \rho$ requires that the inflaton is even lighter [16], and further, that inflation occurring only after stabilization cannot explain the age of the universe 16]. Indeed, the wall-only inflation cannot begin before $t \sim H^{-1} \sim M_{\mathrm{pl}} / M_{*}^{2}>>M_{*}^{-1}$, when the universe is already very large and old.

However, in the models of inflation at very early times which we consider, the effective $4 D$ Planck mass is both much smaller than it is now, and in general, time-dependent due to the variation in the volume of the internal dimensions. Moreover, inflation occurs soon after the "birth" of the universe when the sizes of all dimensions are close to their natural initial size $\sim M_{*}^{-1}$. Hence early asymmetric inflation solves the age problem too.

The framework in which we discuss these issues is that of semiclassical $(4+n)$ dimensional gravity with an additional potential $V(b)$ depending on the size of the internal dimensions. As discussed in Ref. [1 this rapidly becomes a good approximation at energy scales below $M_{*}$, which, self consistently, is the correct regime for early inflation, essentially because of the COBE constraint that the density perturbations are small $\delta \rho / \rho \sim 2 \times 10^{-5}$. Most of our analysis will utilize the so-called string frame with explicit scale factors $(a(t), b(t))$ for the sizes of our brane-localized dimensions and the internal dimensions respectively. In this frame the effective long-distance 4-dimensional value of Newton's constant depends on $b(t)$ and changes with time. However this is the frame where the "measuring sticks" of particle masses and Compton wavelengths are fixed. On the other hand, in the analysis that follows we will find it occasionally useful to employ the correspondence of the low-energy $(4+n)$-dimensional theory with an effective 4-dimensional theory which takes the form of a scalar-tensor theory of gravity, the radius of the large internal space $b$ playing a role similar to the BransDicke scalar field. The inflationary dynamics from the point of view of this frame is completely equivalent to the usual slow-roll scenarios, and the conditions for the 
asymmetric expansion of the universe on the brane relative to the internal dimensions are completely equivalent to the slow roll conditions in the usual inflationary models. Further, this analogy provides very useful consistency check for the determination of the density perturbations. The relationship between the scale factor $b(t)$ and the canonically normalized 4-dimensional ("radion") field is given in equation (23). (We will often abuse our terminology and refer to $b(t)$ itself as the "radion".)

It is important to realize that under quite general conditions, the early inflationary era is followed by a long epoch where the scale factor of our brane-universe undergoes a slow contraction while the internal dimensions continue to expand towards their final stabilized value. We show that even with the inclusion of a potential for $b$, it is possible to exactly describe the evolution during this epoch, and we present a class of exact solutions which generalize the usual vacuum Kasner solutions. The total amount of contraction of our universe on the brane is bounded by a small power of the expansion of the size of internal dimensions, and varies between at most 7 efoldings in the case of two extra dimensions to at most 12 efoldings when there are six extra dimensions. We show that during this phase of $b(t)$ evolution to the stabilization point, the production of bulk gravitons by the time-varying metric remains completely suppressed, ensuring that the bulk is very cold at, and after, the stabilization of the internal dimensions.

However, the particles produced by gravitational (Hawking) effects on the brane at the end of the inflationary de Sitter phase can play an important role. Their energy density is blue-shifted by the slow contraction of $a(t)$, and when this wall-localized energy density exceeds the energy density in the radion, the contraction of $a(t)$ ceases, with a "Big Bounce" occurring, leading on to an expansion of both $a$ and $b$. It is very tempting to view the reheating on the wall as due to this contraction of primordial de Sitter radiation. This is especially so as the final temperatures come naturally close to the normalcy bounds (the upper bound $T_{*}$ on the temperature of [3] that ensure that the evaporation to bulk gravitons remains negligible).

However, such a conclusion is premature, since as we argue below the energy density in the radion is naturally of the same order as that on the wall, and this leads to a radion moduli problem in the later evolution of the universe. Explicitly, because of the gravitationally suppressed couplings of this field to wall-localized SM states, the radion decays very slowly back into light wall fields. As a result, its energy density would red-shift only according to $\rho_{b} \sim 1 / a^{3}$ compared to $\rho_{\text {wall }} \sim 1 / a^{4}$, and comes to dominate the total energy density. This is just the standard moduli problem, which is inextricably linked with the mechanism of reheating in world-as-a-brane models. 
Concretely, the basic picture we advocate is:

- The quantum creation of the universe takes place with the initial size of all dimensions close to the fundamental Planck scale $M_{*}^{-1}$. In particular the initial size $b_{i}$ of the internal dimensions is of this size.

- A prolonged period of inflation in a direction parallel to our brane takes place, with our scale factor $a(t)$ increasing superluminally $a(t) \sim t^{p}$ with $p>>1$, and $b(t)=b_{I}$ essentially static. (In the cases that we explicitly discuss the inflation is quasi-exponential, $a(t) \sim a_{i} \exp (H t)$.) This brane inflation is driven by either the stabilizing potential of the radion $b$ itself, or by a wall-localized field with effective non-zero cosmological constant. In either case it is unnatural for the size of the effective 4-dimensional cosmological constant to exceed roughly $(\mathrm{TeV})^{4}$. Since the internal dimensions are small the effective 4-dimensional Newton's constant is large

$$
G_{N, \text { initial }}=\frac{1}{b_{I}^{n} M_{*}^{n+2}} \simeq \frac{1}{M_{*}^{2}} .
$$

Thus the Hubble constant during this initial period of inflation can be large even though the energy density is quite small, $\langle V\rangle \sim O\left(\mathrm{TeV}^{4}\right)$,

$$
H_{\mathrm{infl}}^{2} \simeq \frac{\langle V\rangle}{b_{I}^{n} M_{*}^{n+2}} \simeq \frac{\langle V\rangle}{M_{*}^{2}}
$$

Thus inflation can be rapid, and moreover, as we will argue in detail the density perturbations can be large, being determined in order of magnitude to be

$$
\frac{\delta \rho}{\rho} \simeq \frac{H_{\text {infl }}}{M_{*}\left(M_{*} b_{I}\right)^{n / 2} S}
$$

where $S$ is a parameter that encapsulates both the duration of $a(t)$ inflation and the deviation of the perturbation spectrum from the scale-invariant HarrisonZeldovich spectrum. (We will argue that $S \lesssim 1 / 50$.) We can turn this around by imposing the COBE-derived normalization on $\delta \rho / \rho$, and thus on $\langle V\rangle / b_{I}^{n} M_{*}^{n+2}$. During this period the size $b_{I}$ of the internal radii are fixed by the over-damping arising from $H_{\text {infl. }}$.

- Wall inflation now ends, with $H$ starting to drop and simultaneously the radion starting to evolve to its' minimum at $b_{0}$. The initial motion of the radion can be quite involved, but from the coupled equations of motion for the scale factors $a(t)$ and $b(t)$ we will see that as $b$ evolves towards its' minimum, our scale factor, under 
very general conditions, undergoes a collapse. The detailed behavior generalizes the well-known Kasner solutions where some dimensions expand, while others collapse, both with determined (subluminal) power-law dependence on time.

- The contraction epoch ends when the blue-shifting radiation density on our brane becomes equal to the energy density in the radion field $\rho_{\text {wall }}=\rho_{b}$. A modelindependent initial source for the radiation on the wall is the Hawking radiation left over from the early inflationary de Sitter phase. After the contraction of $a(t)$ reverses, the radion and wall-localized energy densities scale together until the stabilization point is reached.

- Finally around the stabilization point $b_{0}$ the radion field starts to oscillate freely. Since this energy density scales as $1 / a^{3}$, and the wall-to-radion energy densities are initially comparable at the start of the oscillation era, the radion energy starts to dominate the total energy density.

- The most serious question that early universe cosmology presents in the worldas-a-brane scenario is how do we dilute this energy in radion oscillations to an acceptable level. The radion is long-lived, its' decay width back to light wall states being given by门̆

$$
\Gamma_{\varphi} \simeq \frac{m_{\varphi}^{3}}{M_{\mathrm{pl}}^{2}} .
$$

We thus require some dilution in the radion energy density, either by a short period of late inflation followed by reheating, or by a delayed reheating after $\rho_{b}$ has sufficiently red-shifted. The amount of dilution of the radion energy density that we require is given roughly by $1 \mathrm{eV} / T_{*} \sim 10^{-7}$, so that only about 5 efolds of late inflation is needed.

The paper is organized as follows. In Section 2 we will discuss the details of the inflationary stage, both in the original string (brane) frame and in the effective Einstein frame, showing the initial conditions, the conditions on inflationary potentials and the resolution of the COBE constraints. Section 3 is devoted to the study of the era of radion evolution to stabilization. In subsection 3.1 we derive the exact solutions which generalize simple vacuum Kasner solutions, and which demonstrate that a period of

\footnotetext{
${ }^{\dagger}$ Here $m_{\varphi}$ is the mass of the canonically normalized field corresponding to $b$. The experimental bound on this mass is $m_{\varphi} \gtrsim 10^{-3} \mathrm{eV}$. Note that the decay width is increased if we take into account the possible presence of many branes in the bulk. Indeed some scenarios of radius stabilization e.g., the "brane crystallization" picture [4], require $N_{\text {wall }} \simeq\left(M_{\mathrm{pl}} / M_{*}\right)^{2(n-2) / n}$ branes in the bulk. If each of these have $O(1)$ light modes then the total decay width to all branes is greatly enhanced.
} 
$a(t)$ contraction subsequent to inflation is quite generic. In subsection 3.2 we discuss the phenomenological consequences of this era, including the cause of its cessation and the (minimal) production of bulk gravitons. There we will also show how the normalcy bounds for the temperature of our brane after stabilization are met by the evolution. In subsection 3.3 we discuss we discuss the issue of the production of bulk gravitons during the era of radion evolution and show that the quantities produced are harmless. In subsection 3.4 we provide a physically quite useful discussion of the contraction and stabilization eras in the Einstein frame, where many of the arguments of the previous subsections can be understood quite simply. In subsection 3.5 we provide a short discussion of the radion moduli problem, and quantify its' size using the evolution equations discussed earlier in Section 3. Section 4 gives our conclusions. We also include three extensive Appendices: Appendix A contains a short discussion of some of the basic kinematics of brane evolution embedded in higher dimensions. Appendix B gives some additional details of the exact solutions for the post-inflation slow evolution era where $a(t)$ contracts. Appendix $\mathrm{C}$ proves that the contraction of $a(t)$ is self-ceasing by the blue-shifting of brane-localized radiation, and also discusses the exact solutions that may be obtained for the "Big Bounce" that terminates this contraction.

\section{Early inflation}

We now embark on our detailed discussion of the evolution of the sizes of our brane and the transverse "internal" dimensions. In particular in this section we focus on the physics of the (early) inflationary epoch.

We are interested in the case of a 3 -brane embedded in a $(4+n)$-dimensional spacetime. To reproduce our observed world, ultimately the 3 spatial dimensions parallel to the brane must be as least as large as our current horizon size, while the $n$ transverse spatial dimensions have to stabilize at a size $b_{0}$ given by the constraint on $G_{N}$, (西), $\left(b_{0}\right)^{n} M_{*}^{n+2}=M_{\mathrm{pl}}^{2}$. Note that we take the internal space to be topologically compact from the beginning. That is we impose periodic boundary conditions in the directions transverse to the wall. These conditions reflect the fact that the low energy theory is four-dimensional, and that the classical evolution preserves the topology.

The total action is comprised of a bulk part,

$$
S_{\text {bulk }}=-\int d^{4+n} x \sqrt{-\operatorname{det} G_{(4+n)}}\left(M_{*}^{(n+2)} \mathcal{R}-\mathcal{L}_{\text {matter }}+\ldots\right) \text {, }
$$


and a brane part,

$$
S_{\text {brane }}=-\int d^{4} x \sqrt{-\operatorname{det} g_{(4)}^{\text {induced }}}\left(\mathcal{L}_{\text {standard model }}+\ldots\right)
$$

where $\mathcal{L}_{\text {matter }}$ is the Lagrangian of the bulk fields apart from the graviton. These fields give rise to the stabilizing potential $V$ that we discuss below. The ellipses denote higher-derivative terms that can be safely ignored in most of the regime of interest as curvatures are small compared to the fundamental Planck scale $M_{*}$, apart possibly from the very early pre-inflationary stage immediately after the quantum creation of the universe. However any signatures of such a phase of high energy and large curvature have been wiped out of the visible universe by the subsequent stage of inflation. We will therefore ignore this stage as practically invisible, and begin the description of the world-as-a-brane universe at curvatures an order of magnitude or so below the fundamental Planck scale. Indeed, at and below these scales, the description based on the actions (17) and (8) should be reliable. The background metric for the $(4+$ $n$ )-dimensional spacetime which is consistent with the symmetries of the brane-bulk system is of the form

$$
g_{\mu \nu}=\left(\begin{array}{ccc}
1 & & \\
& -a(t)^{2} g_{I J} & \\
& & -b(t)^{2} g_{i j}
\end{array}\right)
$$

where $a$ is the scale factor of the 3-dimensional space, and $b$ is the scale factor of the internal $n$-dimensional space, with geometry set by $g_{i j}$ where $\operatorname{det}\left(g_{i j}\right)=1$.

As shown in Appendix A, the equations of motion for the coupled $\{a(t), b(t)\}$ system can be written in the form,

$$
\dot{H}_{a}+n H_{a} H_{b}+3 H_{a}^{2}=\frac{1}{2(n+2) b^{n} M_{*}^{(n+2)}}\left(b \frac{\partial V}{\partial b}-(n-2) V\right)
$$

and

$$
\dot{H}_{b}+3 H_{a} H_{b}+n H_{b}^{2}=-\frac{1}{(n+2) b^{n} M_{*}^{(n+2)}}\left(\frac{b}{n} \frac{\partial V}{\partial b}-2 V\right)
$$

where we have introduced the Hubble parameters $H_{a} \equiv \dot{a} / a$ and $H_{b} \equiv \dot{b} / b$ for the two scale factors, and an overdot denotes a derivative with respect to $t$. These equations of motion are supplemented by the constraint equation

$$
6 H_{a}^{2}+6 n H_{a} H_{b}+n(n-1) H_{b}^{2}=\frac{V}{b^{n} M_{*}^{(n+2)}} .
$$


Note that in these formulae the potential $V$ is the effective 4-dimensional potential with mass dimension $[V]=4-i . e$. , that arising from projecting the bulk energy density that results from $\mathcal{L}_{\text {matter }}$ onto our wall.

The natural inflationary initial conditions consist of taking the initial $(4+n)$ dimensional universe to be relatively smooth, flat and potential energy-dominated over scales roughly given by the fundamental Planck length $1 / M_{*}$, and not much greater [19], 20]. In the brane language the initial conditions we adopt mean that we take a portion of the brane of linear size $\sim 1 / M_{*}$ embedded in a $(4+n)$-dimensional volume of the same linear size, to be relatively flat, smooth and straight. Indeed, it is most natural to suppose that the universe starts as a small $(4+n)$-dimensional domain of linear extent $1 / M_{*}$ in all directions, and then undergoes an epoch of inflationary expansion almost immediately, at least in the directions parallel to our brane.

Quantitatively these conditions can be stated in terms of the initial conditions for the brane and bulk horizons as follows. The size of initially isotropic and homogeneous causal domains must be

$$
H_{a, i}^{-1} \sim H_{b, i}^{-1} \geq M_{*}^{-1}
$$

Moreover we can also define the initial conditions for the scale factor of the brane $a$ and the radion field $b$ by referring to the flatness problem. Since close to the fundamental Planck scale the energy density on the wall and in the bulk is of order unity in fundamental Planck units, if we pick the gauge such that any intrinsic spatial curvature on the brane or in the bulk is $k= \pm 1$, it cannot exceed in magnitude $\rho_{p}$, leading to the conditions that

$$
a_{i} \sim b_{i} \geq M_{*}^{-1}
$$

It is easy to recognize the conditions (13) and (14) as precisely the consistent initial conditions for inflation, in the case when the fundamental Planck scale is $M_{*}$. However these initial conditions are merely a rough estimate coming from the requirement that the approximation based on semiclassical gravity is valid, and that the usual cosmological problems (horizon, flatness, homogeneity etc..) are solved by subsequent inflation.

Further, the generation of sufficient density perturbations $\delta \rho / \rho \sim \delta \rho /\left.\rho\right|_{\mathrm{COBE}} \sim 10^{-5}$,

\footnotetext{
${ }^{\ddagger}$ In spatially flat FRW universes the magnitude of the scale factor $a$ is physically meaningless. However, the spatial curvature, namely the quantity $k / a^{2}$ is physical. Thus choosing the value of $a$ at some instant $t$ corresponds to specifying curvature of spatial hypersurfaces. Equivalently, choosing the value of the constant $k$, by normalizing it to \pm 1 , corresponds to picking the units for the scale factors. We will assume this throughout this work, even if we do not explicitly specify the spatial curvatures (which can be ignored after prolonged inflation).
} 
implies, at least without the unappetizing introduction of very light fields on our wall, or of the assumption of very unusual initial conditions, that the inflationary epoch that solves the flatness and horizon problems occurs when the size of the internal dimensions is still relatively small. The basic reason for this is that in a theory with fundamental Planck scale $M_{*}$, the energy density localized on the brane should not exceed $M_{*}^{4}$. Similarly the energy density in the bulk should not exceed $M_{*}^{n+4}$. Moreover, the (matter) energy density of the universe at its' birth is expected to be of order $M_{*}^{n+4}$. If inflation occurs when the internal dimensions are already large, then the effective Newton's constant in our 4-dimensions is already very small $G_{\mathrm{N}, \text { eff }}=1 /\left(M_{*}^{n+2} b^{n}\right) \ll$ $1 / M_{*}^{2}$, and energy densities of order $M_{*}^{4}$ or less will lead to a very small Hubble constant, and thus typically unacceptably small density perturbations. 3

To see this in detail consider the expression for the density perturbations generated during slow-roll inflation driven by a (canonically normalized) field $\varphi$

$$
\frac{\delta \rho}{\rho}=\frac{5}{12 \pi} \frac{H_{\mathrm{inf}}^{2}}{\dot{\varphi}} .
$$

(The use of this 4-dimensional expression will be self-consistently justified later on in our analysis.) The Hubble constant on our brane during inflation is given in order of magnitude by $H_{\text {inf }} \simeq V /\left(M_{*}^{n+2} b_{I}^{n}\right)$, where $b_{I}$ denotes the size of the internal dimensions during the inflationary epoch. Since $V \lesssim M_{*}^{4}$ if $b_{I} M_{*} \gg 1$ then $\delta \rho / \rho$ is very small unless $\dot{\varphi}$ is extremely small. Although this is a logical possibility, it requires extraordinary fine-tuning and we will not consider this case here. On the other hand if $b_{I} M_{*}$ is $\mathcal{O}(1)$ then sufficiently large density perturbations easily result. Moreover, from the power dependence of $H_{\mathrm{infl}}$ on $b_{I}$ we see that if the size of the internal dimensions changes significantly during inflation the spectrum of density perturbations will be very far from scale invariant. Since the spectral index of density perturbations $n_{\rho}$ is constrained by the cosmic microwave background (CMB) and large scale structure measurements to be not significantly different from the scale-invariant value $n_{\rho}=1$,

$$
\left|n_{\rho}-1\right|<0.2
$$

it is necessary that the evolution of the internal dimensions is slow compared to that of our scale factor $a$ : $H_{b} \ll H_{a}$. A successful phenomenology thus results if the ratio $H_{b} / H_{a}$ approaches a zero for a (small) range of $b$ around the value $b_{I}$. Let us expand

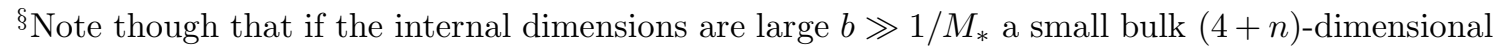
energy density still allows the effective "projected" energy density on the wall to exceed $M_{*}^{4}$, without requiring the use of the full quantum theory of gravity; the semiclassical approximation is still good.
} 
the ratio around this point

$$
\frac{H_{b}}{H_{a}}=S+T\left(\frac{b}{b_{I}}-1\right)^{2}+\ldots
$$

The dimensionless parameters $S$ and $T$ will be bounded in size by the requirements that the spectral index $n$ of the density perturbations is close to 1 , and that sufficient e-folds of inflation occur to solve the horizon and flatness problems. Furthermore $\dot{H}_{b}$ must be small as compared to $H_{a}^{2}$, otherwise the spectral index would again too quickly deviate from 1.

To quantify these restrictions let us return to the equations of motion for $a(t)$ and $b(t)$ and the general expression for the density perturbations. Successful inflation requires that the number of efolds $N_{e}$ of superluminal expansion be sufficiently large. If the initial and final values of the scale factors just before and just after the inflationary epoch are denoted by subscripts $(i, f)$ respectively, then

$$
\begin{aligned}
N_{e} & =\int_{a_{i}}^{a_{f}} \frac{d a}{a} \\
& =\int_{b_{i}}^{b_{f}} \frac{H_{a}}{H_{b}} \frac{d b}{b} \\
& \simeq \int_{b_{i}}^{b_{f}} \frac{1}{S+T\left(b / b_{I}-1\right)^{2}} \frac{d b}{b} .
\end{aligned}
$$

To a good approximation inflation begins and ends when the condition $H_{b} / H_{a} \ll 1$ is violated. Hence to obtain inflation, there are two possibilities. Either $S$ must satisfy $S \ll 1$, which in turn implies

$$
\frac{b_{i, f}}{b_{I}}=1 \mp T^{-1 / 2}
$$

The alternative is to have $S$ small (but not excessively) while $T$ must be extremely small, which corresponds to slow, power law, inflation. Substituting these endpoint values into (19) and performing the integral gives the constraint on $S$ and $T$ arising from $N_{e}$ :

$$
N_{e} \simeq \frac{1}{S+T}\left(\frac{2 \sqrt{T} \tan ^{-1}(1 / \sqrt{S})}{\sqrt{S}}-\log (1+1 / S)+2 \log (1+1 / \sqrt{T})\right)
$$

Requiring, say, $N_{e}>100$ then puts an upper bound on the size of $(S, T)$. In various limits this relation becomes easy to state explicitly. For example if $S=T \ll 1$ then

$$
N_{e} \simeq \frac{\pi}{2 T}
$$


while if $S$ is very small, while $T$ remains $O(1)$,

$$
N_{e} \simeq \frac{\pi}{\sqrt{S}}-\log (1 / S)
$$

In any case obtaining $N_{e} \sim 100$ efolds of inflation requires either $S \simeq T \lesssim 0.02$, or $T \simeq O(1)$, and $S \lesssim 10^{-3}$. So in practice to get a sufficient number of efolds $S$ should be somewhere in the range $0.001 \lesssim S \lesssim 0.02$ (or, of course less) depending on $T$.

Now let us turn to the constraint arising from the magnitude and spectral index of the density perturbations. To do this in detail, we must make some assumptions about the identity of the inflaton. Since we have argued that inflation should occur far away from the eventual stabilization value of $b$, the most natural candidate for the potential energy that drives inflation is the radion potential itself, with therefore the radion playing the role of the inflaton. The correctly normalized field $\varphi$ corresponding to the scale factor $b$ is given by (see Appendix A)

$$
\dot{\varphi}=\sqrt{2 n(n-1)} M_{*}^{(n+2) / 2} b^{(n-2) / 2} \dot{b}
$$

Substituting this into the expression for the density perturbations (15) we find

$$
\begin{aligned}
\frac{\delta \rho}{\rho} & =\frac{5}{12 \pi \sqrt{2 n(n-1)}} \frac{H_{a}^{2}}{M_{*}^{(n+2) / 2} b^{(n-2) / 2} \dot{b}} \\
& \simeq \frac{5}{12 \pi \sqrt{2 n(n-1)}} \frac{H_{a}}{M_{*}\left(M_{*} b_{I}\right)^{n / 2} S},
\end{aligned}
$$

where in the last step we have used (17) and the fact that we are close to $b_{I}$. Requiring $b(t)$ to be essentially static during inflation, and in particular that $H_{b} / H_{a}$ and $\dot{H}_{b} / H_{a}^{2}$ are small near $b_{I}$ is equivalent, using the equations of motion, to the statement that

$$
\left.\frac{2\left(\frac{b}{n} \partial_{b} V-2 V\right)}{3\left(b \partial_{b} V-(n-2) V\right)}\right|_{b \simeq b_{I}} \ll 1 .
$$

which translates into the condition that

$$
\left.\left(\frac{b}{n} \partial_{b} V-2 V\right)\right|_{b_{I}} \ll V\left(b_{I}\right), \quad b_{I} \partial_{b} V\left(b_{I}\right) .
$$

Therefore $\left.\left.\left(b \partial_{b} V\right)\right|_{b_{I}} \simeq 2 n V\right|_{b_{I}}$, and up to small corrections, the Hubble parameter $H_{a}$ during inflation is given by

$$
H_{a}^{2} \simeq \frac{V\left(b_{I}\right)}{6 b_{I}^{n} M_{*}^{(n+2)}} .
$$


Finally this together with (25) gives the expression for the inflation generated density perturbations

$$
\frac{\delta \rho}{\rho} \simeq \frac{5}{12 \pi} \frac{1}{S\left(M_{*} b_{I}\right)^{n}}\left(\frac{V\left(b_{I}\right)}{12 n(n-1) M_{*}^{4}}\right)^{1 / 2} .
$$

The spectral index $n_{\rho}$ is defined by the comoving wavenumber dependence

$$
\frac{\delta \rho}{\rho} \sim k^{\left(n_{\rho}-1\right) / 2}
$$

where at horizon crossing we have the relation $k / a=H_{a}$. From (29) we can extract a more convenient expression for $n_{\rho}$ :

$$
\begin{aligned}
n_{\rho}-1 & =2 \frac{d \log (\delta \rho / \rho)}{d \log (a)} \\
& \simeq 2 S \frac{d \log (\delta \rho / \rho)}{d \log (b)},
\end{aligned}
$$

where in the second line the parameterization for $H_{b} / H_{a}$ (17) has been used. Applying this formula to the expression for the density perturbations (28) leads to

$$
n_{\rho}-1=2 S\left(-n+\frac{1}{2 V} \frac{d V}{d \log (b)}\right) .
$$

But from the two expressions for the ratio $H_{b} / H_{a}$, one from the equations of motion (10,11), and the other given by the parameterization (17) we have, after some algebra,

$$
\frac{1}{2 V} \frac{d V}{d \log (b)}=n-\frac{n(n+2)}{4}\left(S+T\left(b / b_{I}-1\right)^{2}\right)
$$

and therefore

$$
n_{\rho}-1 \simeq-\frac{n(n+2)}{2}\left(S^{2}+S T\left(b / b_{I}-1\right)^{2}\right)
$$

This expression together with the experimental constraint $\left|n_{\rho}-1\right|<0.2$ forces $S \lesssim 0.1$, thus ruling out the case of $T \ll 1, S \sim O(1)$ allowed by the earlier condition of a sufficient number of efolds of inflation. Note however that the solutions with $T \sim O(1)$ and $S \lesssim 0.01$ automatically satisfy $\left|n_{\rho}-1\right|<0.2$ over essentially the whole range of $a(t)$ inflation. (Experimentally all we require is that $\left|n_{\rho}-1\right|<0.2$ in the range of scales between the COBE and large-scale structure measurements - roughly 10 efolds rather than the full duration of inflation.)

Finally we can use the magnitude of the measured CMB fluctuations to constrain the size $b_{I}$ of the internal dimensions during the inflationary epoch. Recall that COBE 
and the other CMB measurements tell us that at the time the scales $k \simeq 7 H_{\text {now }}$ were being inflated outside of the horizon, the density perturbations were of size

$$
\left.\frac{\delta \rho}{\rho}\right|_{\mathrm{COBE}} \simeq 2 \times 10^{-5} \text {. }
$$

This together with the formula for $\delta \rho / \rho(28)$, and the bound on the parameter $S$ leads to a constraint on a combination of the size of the potential during inflation $V\left(b_{I}\right) / M_{*}^{4}$ and the volume $V_{I}=\left(b_{I}\right)^{n}$ of the internal dimensions, both in units of the fundamental Planck scale:

$$
V_{I} M_{*}^{n} \simeq 5 \times 10^{4}\left(\frac{0.02}{S}\right)\left(\frac{V\left(b_{I}\right)}{M_{*}^{4}}\right)^{1 / 2} .
$$

For $S \simeq T \simeq 0.02$ and $V\left(b_{I}\right)$ having a perfectly reasonable value of $V\left(b_{I}\right) \simeq(200 \mathrm{GeV})^{4}$, (for example if $M_{*} \simeq 1 \mathrm{TeV}$ ) we therefore discover that the epoch of inflation generating COBE needs to occur around the value $b_{I} \simeq 10^{3 / n} M_{*}^{-1}$, i.e., when the internal dimensions are still relatively small as expected.

Now let us return to the justification of the use of the usual 4-dimensional expression (15) for the density perturbations. If the de Sitter horizon $\left(H_{a}\right)^{-1}$ during inflation is much smaller than the size of the internal dimensions then the full $(4+n)$-dimensional expression for the density fluctuations must be employed, while if $\left(H_{a}\right)^{-1} \gg b_{I}$ then it is correct to use the long-distance, effectively 4-dimensional, expression. From the

constraint arising from $\delta \rho / \rho$ (35), and the expression for $H_{a}$ during inflation (27) we find that

$$
H_{a} b_{I} \simeq \frac{\left(5 \times 10^{-3}\right)^{(n-2) / n}}{\sqrt{6}}\left(\frac{S}{0.02}\right)^{(n-2) / 2 n}\left(\frac{V\left(b_{I}\right)}{M_{*}^{4}}\right)^{(n+2) / 4 n} .
$$

Thus $H_{a} b_{I}$ is always substantially less than 1 for the range of parameters of interest, and the 4 -dimensional description of the generation of $\delta \rho / \rho$ is correct.

\subsection{Inflation from the Einstein frame perspective}

Most dynamical aspects the world-as-a-brane scenario are the most transparent in terms of the geometric variables employed so far, which can be referred to as the string-frame quantities. The reason is that the kinematics in this frame is automatically expressed in terms of the units felt by the observers which live on the wall. In particular, such observers choose to define scales using their own masses and Compton wavelengths as yard sticks, and in the string frame these yard sticks are time independent. However, it is illustrative to consider geometrical evolution of the universe in light of the reference frame where the gravitational sector of the theory coincides with classical Einstein 
gravity. In this frame, the equations of motion resemble the coupled gravity-matter models considered in the context of non-minimal theories of gravity [21], and the results obtained from them can be a useful addition to physical intuition.

For this reason, here we will recast the description of the inflationary stage into the Einstein conformal frame of the theory on the brane (17), (8). This frame is defined by the requirement that the propagator of the graviton is canonical. The map which casts the kinetic term of the graviton into the canonical form is

$$
\bar{g}_{\mu \nu}=\left(\frac{M_{*}}{M_{\mathrm{pl}}}\right)^{2}\left(M_{*} b\right)^{n} g_{\mu \nu}
$$

Explicitly, we can define the Einstein frame comoving time and scale factor according to

$$
d \bar{t}=\frac{M_{*}}{M_{\mathrm{pl}}}\left(M_{*} b\right)^{n / 2} d t \quad \bar{a}=\frac{M_{*}}{M_{\mathrm{pl}}}\left(M_{*} b\right)^{n / 2} a
$$

where all barred quantities refer to the Einstein frame. Now, the radion field is equivalent to a scalar field, defined by

$$
M_{*}^{n+2} b^{n}=M_{\mathrm{pl}}^{2} \exp \left(-\sqrt{\frac{n}{2(n-1)}} \frac{\varphi}{M_{\mathrm{pl}}}\right)
$$

Note that the normalization employed here is dictated by the definition of the perturbation of the radion away from its mean value during inflation, which sets the effective background Planck mass. We note that the initial conditions for inflation in the Einstein frame, in the units of the effective Planck scale, require homogeneity and flatness over distances of $\sim l_{P l, e f f}=1 / M_{\mathrm{pl}}$, which can be recognized as a usual inflationary initial condition.

This Einstein frame picture is very useful to compute the density contrast in models where radion is the inflaton. The density contrast is given by the standard formula

$$
\frac{\delta \rho}{\rho}=\frac{\bar{H}^{2}}{2 \pi \varphi^{\prime}}=8 \frac{\bar{H}^{2}}{\varphi^{\prime}}
$$

in our normalizations, and where the prime is the derivative with respect to $\bar{t}$. To determine the density contrast in the string frame, we recall that it is, roughly, conformally invariant during inflation [22], and conformally transform (40) to the string frame. The radion and the Hubble parameter transform to

$$
\begin{aligned}
\varphi^{\prime} & =-\sqrt{2 n(n-1)} \frac{M_{\mathrm{pl}}^{2} H_{b}}{M_{*}\left(M_{*} b\right)^{n / 2}} \\
\bar{H} & =\frac{M_{\mathrm{pl}}}{M_{*}\left(M_{*} b\right)^{n / 2}}\left(H_{a}+\frac{n}{2} H_{b}\right)
\end{aligned}
$$


which is straightforward to determine from their definitions. With this, we find

$$
\frac{\delta \rho}{\rho}=\frac{8}{\sqrt{2 n(n-1)}} \frac{\left(H_{a}+\frac{n}{2} H_{b}\right)^{2}}{M_{*}\left(M_{*} b\right)^{n / 2} H_{b}}
$$

Now we consider the tilt of the perturbation spectrum. To consider it, we define the effective slope by

$$
\theta \sim \frac{\frac{\delta \rho}{\rho}\left(t_{a}\right)}{\frac{\delta \rho}{\rho}\left(t_{b}\right)} \sim\left(\frac{b\left(t_{b}\right)}{b\left(t_{a}\right)}\right)^{n / 2}
$$

where the last equality arises because of the slow roll conditions $H_{a, b}\left(t_{a}\right) \sim H_{a, b}\left(t_{b}\right)$ during inflation. Since $b\left(t_{a}\right) \sim b\left(t_{b}\right)\left(1+H_{b} \delta t\right)$ and since we compare the tilt between 50 and 60 efoldings, we get $\delta t \sim 10 H_{a}^{-1}$. Thus roughly, $\theta \sim 1-5 n \frac{H_{b}}{H_{a}}$ On the other hand, during this time $\theta$ changes according to $\theta \sim\left(\frac{k_{a}}{k_{b}}\right)^{\left(n_{\rho}-1\right) / 2}$, and given that $k_{k} \sim a_{k} H_{a}$, we find that the ratio of the wave vectors is given by $k_{a} / k_{b} \sim a_{a} / a_{b} \sim \exp \left(H_{a} \delta t\right) \sim \exp (10)$. Taking this and the bounds on the spectral index $n_{\rho}, n_{\rho} \leq 1 \pm 0.2$, we find that

$$
\theta \sim e^{ \pm 1 / 2}
$$

This leads to the following inequality which the expansion rates must satisfy during inflation:

$$
H_{a} \gtrsim \frac{5 n}{1-\exp ( \pm 1 / 2)} H_{b} \geq 15 n H_{b}
$$

As a result, the density contrast $\delta \rho / \rho$ is

$$
\frac{\delta \rho}{\rho} \simeq \frac{8}{\sqrt{2 n(n-1)}} \frac{H_{a}^{2}}{M_{*}\left(M_{*} b\right)^{n / 2} H_{b}}
$$

The COBE data tell us that $\delta \rho / \rho \sim 10^{-5}$. Using this and $H_{a} \geq 15 n H_{b}$, we find

$$
H_{a} \lesssim 10^{-7} M_{*}\left(M_{*} b\right)^{n / 2}
$$

We can use this inequality to obtain a bound on the size of the internal dimensions $b_{I}$ during inflation. We repeat that the evolution is similar to the Einstein frame thanks to the slow roll conditions. Hence the vacuum energy density during inflation is

$$
\rho_{\text {vacuum }} \sim M_{*}^{(2+n)} b_{I}^{n} H_{a}^{2}
$$

Using our estimate for $H_{a}$,

$$
\rho_{\text {vacuum }} \sim 10^{-14} M_{*}^{4}\left(M_{*} b_{I}\right)^{2 n}
$$


However $\rho_{\text {vacuum }}$ must not exceed $M_{*}^{4}$. In fact, since initially the theory is very close to the quantum gravity scale, in order to insure the validity of the semiclassical approximation the energy density cannot exceed, let's say, $10^{-5} M_{*}^{4}$, meaning that the energy scale is about a factor of $10^{5 / 4}$ below the quantum gravity scale. This ensures that the semiclassical description is correct. Using the equation for the energy density in terms of $b_{I}$ above, we obtain the inequality

$$
b_{I} \lesssim 10^{9 / 2 n} M_{*}^{-1}
$$

Note that the upper limit on $b_{I}$ ranges between about $200 M_{*}^{-1}$ for $n=2$ to about $6 M_{*}^{-1}$ for $n=6$. In either case however, these are clearly the correct initial conditions for inflation, which in fact come naturally in this context.

\section{Post-inflation evolution to stabilization point}

As we have discussed in the previous section, when the inflationary stage ends, the ratio of internal space to on-brane Hubble rates of expansion $H_{b} / H_{a}$ approach unity. From this time on, the slow roll conditions for the effective potential cannot be upheld any more. The kinetic energy stored in the expansion of the internal space begins to play a significant role in the evolution of the brane-world. Rather interestingly, however, we will see that under rather general conditions the expansion of the whole higherdimensional universe under the combined influence of the potential and kinetic energies during this era is well approximated by a generalization of the well-known Kasner solutions. These solutions generically describe cosmic evolution which is anisotropic in different directions, with a subset of the directions contracting while the others expand. Specifically we will find that after the end of the initial brane-world inflation the directions longitudinal to the brane contract, while the internal directions expand, both according to some power-law time dependence. The precise form of the power laws is controlled by the codimension of the brane ( $n$ in our notation) and the leadingorder behavior of the stabilizing potential $V(b)$ as a function of the scale factor $b$ of the internal dimensions.

\subsection{Theory of the era of contraction}

To illustrate generic features of such behavior, and show why it should be immediately expected, at least in a subset of cases, consider the limiting case where after the exit from the inflationary stage the potential $V(b)$ drops by many orders of magnitude. 
The evolution of the scale factors $a(t)$ and $b(t)$ is then controlled entirely by the kinetic energy, and the equations of motion appropriate for this case are just the higherdimensional vacuum Einstein equations,

$$
R_{\mu \nu}=0
$$

In other words (10,11) with $V$ set to zero. The $(4+n)$-dimensional solutions which are consistent with the brane symmetries are of the form

$$
d s^{2}=-d t^{2}+a_{i}^{2}\left(\frac{t}{t_{i}}\right)^{2 k} d \vec{x}_{3}^{2}+b_{i}^{2}\left(\frac{t}{t_{i}}\right)^{2 \ell} d \vec{y}_{n}^{2}
$$

where $a_{i}, b_{i}$ are now the "initial" values of the scale factors as set by the end of the inflationary stage. The powers $k$ and $\ell$ are uniquely determined by the Einstein equations, which reduce to two simple algebraic equations,

$$
\begin{aligned}
3 k+n \ell & =1 \\
3 k^{2}+n \ell^{2} & =1
\end{aligned}
$$

A metric of the form (52) with exponents satisfying (53) is known as the Kasner solution. The solutions of the algebraic equations for the exponents are

$$
\begin{aligned}
& k=\frac{3 \mp \sqrt{3 n(n+2)}}{3(n+3)} \\
& \ell=\frac{n \pm \sqrt{3 n(n+2)}}{n(n+3)}
\end{aligned}
$$

Phenomenologically, we certainly need the internal dimensions to grow in size and approach the stabilizing value, and this selects the upper sign in the equalities (54), which then implies that our longitudinal brane directions contract. As we will soon see when we re-introduce the potential $V(b)$, this behavior is physically selected by the asymmetry embodied in the potential, and in particular the fact that it must have a minimum at the stabilizing value $b_{0}$, with $b_{0}^{n}=M_{\mathrm{pl}}^{2} / M_{*}^{n+2}$. In any case in this simple potential-free Kasner case, the explicit values for the powers range between $k=-0.1266, \ell=0.69$ for $n=2$, and $k=-1 / 3, \ell=1 / 3$ for $n=6$.

It is useful to express the brane scale factor $a(t)$ as a function of the size of the internal dimensions $b(t)$,

$$
\frac{a}{a_{i}}=\left(\frac{b}{b_{i}}\right)^{-|k| / \ell}
$$


The amount of contraction of our scale factor is thus determined by the increase in $b$ (which in turn is determined once the end of inflation value of $b=b_{i}$ is specified), and the known power $-|k| / \ell$. Note that for $n=2, k / \ell \sim-0.183$, and so the brane world contracts by only one order of magnitude for almost six orders of magnitude of radion increase. As $n$ increases, this dependence speeds up, and for $n=6$ the contraction of the brane dimensions and expansion of the internal dimensions are essentially equal in magnitude (we have of course ignored small transient effects at both the start and end of this Kasner phase which slightly modify the above relationships).

We are typically interested, however, in the case where there exists a non-trivial $V(b)$ potential as well as matter on our brane. We again take the metric to be of the form

$$
d s^{2}=-d t^{2}+a^{2}(t) d \vec{x}_{3}^{2}+b^{2}(t) d \vec{y}_{n}^{2}
$$

and assume that the matter on the wall is represented by a classically conserved, perfect fluid energy-momentum tensor $T_{\mu \nu}=(\rho+p) u_{\mu} u_{\nu}+p g_{\mu \nu}, \nabla_{\mu} T^{\mu \nu}=0$, where $u_{\mu}$ is a future-oriented timelike vector, with the components $u^{\mu}=\left(1, \overrightarrow{0}_{3+n}\right)$ in the basis (56). Here $\rho$ is the energy density of the wall matter and $p=\gamma \rho$ is the pressure, with $\gamma$ a constant given by the speed of sound on the wall. The equations of motion slightly generalize (10) and (11),

$$
\begin{aligned}
& 6 H_{a}^{2}+n(n-1) H_{b}^{2}+6 n H_{a} H_{b}=\frac{V+\rho}{M_{*}^{n+2} b^{n}} \\
& \frac{\ddot{b}}{b}+(n-1) H_{b}^{2}+3 H_{b} H_{a}=\frac{1}{M_{*}^{n+2} b^{n}}\left(\frac{2 V}{n+2}-\frac{b}{n(n+2)} \frac{\partial V}{\partial b}+\frac{\rho-3 p}{2(n+2)}\right) \\
& \frac{\ddot{a}}{a}+2 H_{a}^{2}+n H_{b} H_{a}=\frac{1}{M_{*}^{n+2} b^{n}}\left(\frac{b}{2(n+2)} \frac{\partial V}{\partial b}-\frac{n-2}{2(n+2)} V+\frac{\rho+(n-1) p}{2(n+2)}\right) \\
& \dot{\rho}+3 H_{a}(p+\rho)=0 .
\end{aligned}
$$

The last equation is of course the usual wall energy-momentum conservation equation, $\nabla_{\mu} T^{\mu \nu}=0$.

It is important to note that the contraction of the brane in Kasner-like solutions leads to an increase of the energy density of any matter or radiation present on the brane at the beginning of the contraction, and this will lead to "bounce" solutions that will be important to us later. Also note that these equations generically receive quantum corrections from particle production via curved space effects or the conformal anomaly. The corrections would manifest themselves as a nonzero source term on the RHS, which as we will see would lead to significant effects only early on, and therefore could be modeled by an appropriate choice of the initial condition for $\rho$. 
Namely, the energy density on the wall corresponds to the particles produced by changing gravitational fields at the end of inflation. Since these phenomena are essentially similar to Hawking radiation, the value of $\rho$ as compared to the potential is suppressed by a factor of $H_{a}^{2} / M^{n+2} b^{n}$ at the end of inflation. Using the COBE data to constrain this quantity, we see that initially $\rho$ is much smaller than $V$. Hence ignoring it is an excellent approximation. For most of this stage, therefore, the dynamics of the universe is determined by the interplay of the radion kinetic and potential energy, which completely determine the evolution of the wall geometry. We will simply carry on with the analysis of (57), treating $\rho$ as a small perturbation and ignoring its backreaction on $a$ and $b$. However, although to the lowest order, the particle production phenomena are negligible, they could lead to interesting effects for reheating. We will return to this later.

However before we consider such blue-shifting of the brane-localized energy density, let us turn our attention to the more general and appropriate case of non-negligible radion potential. Remarkably in this case a form of Kasner-like behavior still applies.

Quite generically, in the semiclassical limit, fully valid at this stage of the evolution, the potential may be viewed as an expansion in inverse powers of the radion field $b$. At generic values of $b$ away from the stabilization point the potential will be dominated by a single term in this expansion. Hence, we can simply approximate $V(b)$ by a monomial of the form $V=W b^{-p}$, where $W$ is a dimensionful parameter with $[W]=4-p$.

If we substitute $V=W b^{-p}$ in the equations of motion, ignoring $\rho$ and $p$ for the moment we find

$$
\begin{aligned}
& 6 H_{a}^{2}+n(n-1) H_{b}^{2}+6 n H_{a} H_{b}=\frac{W}{M_{*}^{n+2} b^{n+p}} \\
& \frac{\ddot{b}}{b}+(n-1) H_{b}^{2}+3 H_{b} H_{a}=\frac{(2 n+p) W}{n(n+2) M_{*}^{n+2} b^{n+p}} \\
& \frac{\ddot{a}}{a}+2 H_{a}^{2}+n H_{b} H_{a}=-\frac{(n+p-2) W}{2(n+2) M_{*}^{n+2} b^{n+p}} .
\end{aligned}
$$

These equations can in fact be solved exactly! With appropriate field redefinitions and gauge (coordinate) transformations, they can be mapped to a system of equations describing the motion of two particles in one dimension, one free, and another Liouville with an exponential potential.

First note that in a certain parameter range these exact solutions asymptotically

\footnotetext{
${ }^{\AA} W$ could well depend logarithmically on $b$. This mild additional $b$ dependence will not change either our qualitative, or to a good approximation our quantitative conclusions. For simplicity we ignore it in the following discussion.
} 
converge to the "potential-free" Kasner solutions (52) and (54) above, as we discuss in Appendix B. This parameter range turns out to be the one in which, upon substitution of the power dependence (54) into the equations (58), the potential terms on the RHS vanish more quickly as a function of time than the LHS. Since the LHS always scales as $\sim t^{-2}$ this is the case when $(n+p) \ell>2$, or equivalently

$$
\frac{(n+p)}{n(n+3)}(n+\sqrt{3 n(n+2)}>2
$$

This gives a curve in $(n, p)$ space above which (i.e., for larger values of $p)$ the exact solution asymptotes to the potential-free Kasner exponents. The critical values of $p$ vary from 0.899 at $n=2$ to $p=0$ at $n=6$.

We now discuss the changes of variables which allow the exact solution of the equations with potential. First define

$$
a=a_{i} e^{\alpha(t)} \quad b=b_{i} e^{\beta(t)}
$$

where $a_{i}$ and $b_{i}$ are as before the initial values of the scale factors at the beginning of the epoch of radion coasting. This means that the appropriate initial conditions for the dimensionless variables $\alpha$ and $\beta$ are $\alpha_{i}=\beta_{i}=0$. Further, defining the parameter $\omega=\frac{W}{M_{*}^{n+2} b_{i}^{n+p}}$, substituting this and (60) into (58), and going to the new time variable $\tau$ :

$$
d \tau=-e^{-3 \alpha-n \beta} d t
$$

leads after some simple algebra to the equations of motion in their final form, suitable for explicit analysis:

$$
\begin{aligned}
& 6 \alpha^{2}+n(n-1) \beta^{2}+6 n \alpha^{\prime} \beta^{\prime}=\omega e^{6 \alpha+(n-p) \beta} \\
& \beta^{\prime \prime}=\frac{(2 n+p) \omega}{n(n+2)} e^{6 \alpha+(n-p) \beta} \\
& \alpha^{\prime \prime}=-\frac{(n+p-2) \omega}{2(n+2)} e^{6 \alpha+(n-p) \beta}
\end{aligned}
$$

Here primes denote derivatives with respect to $\tau$. The specific form of $\tau=\tau(t)$ can be determined after the solutions are found.

The equations (62) are immediately integrable. Indeed, consider the linear combination $4 \alpha+\frac{2 n(n+p-2)}{2 n+p} \beta$. By using the second order differential equations, it is easy to verify that $4 \alpha^{\prime \prime}+\frac{2 n(n+p-2)}{2 n+p} \beta^{\prime \prime}=0$. Hence we can immediately write one first integral of $(62)$ :

$$
4 \alpha+\frac{2 n(n+p-2)}{2 n+p} \beta=C_{1}+C_{2} \tau
$$


where $C_{1}$ and $C_{2}$ are integration constants to be determined later.

To find the other integral of motion, we can define the new variable

$$
X=6 \alpha+(n-p) \beta
$$

The remaining independent second order differential equation becomes

$$
X^{\prime \prime}=\frac{6 n-4 n p-n^{2}-p^{2}}{n(n+2)} \omega e^{X}
$$

This is the Liouville equation, corresponding to a particle in 1 dimension moving in an exponential potential. This has an easily determined first integral, simply given by the conservation of energy:

$$
X^{\prime 2}=2 \frac{\Delta}{n(n+2)} \omega e^{X}+E_{0}
$$

with $E_{0}$ the energy integral, and where we have defined $\Delta=6 n-4 n p-n^{2}-p^{2}$. At this moment, we need to make three observations. First, $X$ is a good independent variable only as long as $\Delta$ is nonzero. If it is zero, the variable $X$ degenerates to the previous first integral (63), up to an overall constant, and so another independent integral should be used. This is easy to take into account however, and besides corresponds to a set of measure zero in the phase space of solutions, and thus we will not pay it much attention here. We will instead focus on the more generic cases where $X$ is independent from (63).

Second, the curve in the $(n, p)$ plane where $\Delta=0$ vanishes is precisely the curve defined by (59) that separates the traditional potential-free Kasner solutions from the more general (but still asymptotic power-law) behavior that we discuss below. In particular, the traditional potential-free Kasner solutions apply asymptotically in the region $\Delta<0$. The full behavior in this region, including the transient regime before the power-law dependence on $t$ sets in can easily be discussed by a simple generalization of the analysis described below and in Appendix $\mathrm{B}$ for the case $\Delta>0$. Leaving the region $\Delta<0$ to the Appendix, we focus on the novel case of $\Delta>0$ in the following.

Third, the integrals of motion $C_{2}$ and $E_{0}$ are not independent by virtue of the Einstein constraint equation, which is the first equation of (62). If we take (66) and the first derivative of (63), and substitute them into the constraint, we find

$$
E_{0}=\frac{3(2 n+p)^{2}}{4 n(n+2)} C_{2}^{2}
$$


Thus we see that the constant $C_{2}$ completely controls the dynamics. With this, we have essentially reduced the system (62) to a functional constraint (63) and a simple $1^{\text {st }}$ order equation

$$
X^{\prime 2}=2 \frac{\Delta}{n(n+2)} \omega e^{X}+\frac{3(2 n+p)^{2}}{4 n(n+2)} C_{2}^{2}
$$

which can be easily integrated when $\Delta \neq 0 . \|$

As shown in Appendix B when $\Delta>0$, the exact solutions of these equations yield the long-time behavior of the scale factors $a(t)$ and $b(t)$. It is given by

$$
\begin{aligned}
& a=a_{i}\left(\frac{t}{t_{i}}\right)^{-\frac{n(n+p-2)}{(n+p)(2 n+p)}} \\
& b=b_{i}\left(\frac{t}{t_{i}}\right)^{\frac{2}{n+p}}
\end{aligned}
$$

These solutions describe a situation in which the on-brane scale factor shrinks while the size of the internal dimensions continues to grow. The exact solutions show that for $C_{2}=0$ they are generally valid, while they are asymptotic long-time attractors for the generic cases with $C_{2}<0$ (i.e., the case in which both $H_{a}$ and $H_{b}>0$ after the end of inflation). This means that even if the brane scales initially continue to expand, soon after the end of inflation they inevitably start to contract. Note that the $t$-dependence of $b(t)$ is exactly such as to have the potential-dependent RHS of the evolution equations scale in the same $1 / t^{2}$ fashion as the LHS.

In any case, the main point of this analysis is that the evolution after the initial stage of inflation continues into a phase of slow progress of $b(t)$ towards the stabilization point, with, under quite general conditions, a simultaneous contraction of our brane scale factor $a(t)$.

The amount of $a(t)$ contraction is controlled by the exponents in (69) (or in the case of $\Delta<0$ the exponents in (54)), and the amount of expansion of $b$ until the stabilization point is reached. We can use the asymptotic form of the solutions to place an upper bound on the amount of contraction of the brane as a function of the evolution of $b$. We have

$$
\frac{a}{a_{i}} \leq\left(\frac{b_{i}}{b}\right)^{\zeta}
$$

where the parameter $\zeta$ is given by

$$
\zeta=\frac{n(n+p-2)}{2(2 n+p)} \quad \text { for } \Delta>0
$$

\footnotetext{
"If $\Delta=0$ a simple modification of this procedure still yields the second integral of motion.
} 


$$
\zeta=\frac{3 n-\sqrt{3 n(n+2)}}{6} \quad \text { for } \Delta<0 .
$$

In these equations recall that $\Delta=6 n-4 n p-n^{2}-p^{2}$, while the effective 4-dimensional potential for $b$ varies as $V \sim b^{-p}$. It is easy to see that the exponents for the $a(t)$ and $b(t)$ evolution calculated in the $\Delta>0$ and $\Delta<0$ regions are continuous on the curve $\Delta=0$, and thus so is $\zeta$. Furthermore, note that for a given $n$ the greatest $a(t)$ contraction occurs in the $\Delta<0$ case.

\subsection{Phenomenology of the era of contraction}

There are a number of interesting consequences of the period of $a(t)$ contraction just described. First, during the contraction era, the brane universe looses a number of efoldings of the scale factor to the shrinkage. This implies that the early period of inflation needs to produce that many efoldings more than the naive minimum needed to solve the horizon and flatness problems. Moreover, the contraction of $a(t)$ blueshifts any energy density left on our wall at the end of inflation, and as we will argue below, this could, together with the remnant of the de Sitter era Hawking radiation, conceivably be the source of reheating of our brane.

To quantify these remarks, we note that the contraction factor of the brane cannot exceed the amount

$$
\frac{a_{f}}{a_{i}} \leq\left(\frac{b_{i}}{b_{0}}\right)^{\zeta}
$$

where the fact that the final value of $b(t)$ is well-approximated by the stabilizing value, $b_{0}$, has been used. The value of $b_{i}$ is set by the size of the internal dimensions at the end of the early period of inflation and at worst is of order the fundamental Planck length $b_{i} \geq M_{*}^{-1}$. (The COBE constraint (35) typically requires a larger value of $b_{i}$, and this just lessens the amount of contraction.) Thus, the maximum amount of contraction is bounded from above by

$$
\frac{a_{f}}{a_{i}} \leq\left(\frac{M_{*}}{M_{\mathrm{pl}}}\right)^{2 \zeta / n} .
$$

The numerical value of this formula ranges between about 900 for $n=2$ to about $2 \times 10^{5}$ for $n=6$. This means that we loose to contraction at most about 7 efoldings of inflation for $n=2$ up to about 12 for $n=6$. Clearly, this is not excessive, and can be easily made up for by a slightly longer period of early inflation.

We now turn to the evolution of the wall and bulk energy densities during the period of contraction. First note that the dominant form of energy density at the end of the 
inflationary period is the bulk energy of the radion, i.e.the $b(t)$ scale factor kinetic and potential energy. The (effective 4-dimensional) kinetic energy density of the radion is

$$
\rho_{b, K E}=n(n-1) M_{*}^{n+2} b^{n}\left(H_{b}\right)^{2}
$$

while its 4-dimensional potential energy density is just $V(b)$ as used in the previous sections. ¿From the exact solutions presented in the previous subsection we find that scaling of the energy density of the radion depends on the exponents $(k, \ell)$ for the evolution of the scale factors $a \sim t^{k}$, and $b \sim t^{\ell}$, which in turn depend on whether the potential is important or not, in other words the sign of $\Delta$. The result is

$$
\begin{array}{ll}
\Delta \geq 0 & \text { (potential case) } \\
& \rho_{b, P E} \sim b^{-p}, \quad \rho_{b, K E} \sim b^{-p} \\
\Delta<0 \quad & \text { (simple Kasner) } \\
& \rho_{b, P E} \sim 0, \quad \rho_{b, K E} \sim b^{2 n-\sqrt{3 n(n+2)}} .
\end{array}
$$

So far we haven't discussed what ends the contraction period. As shown in detail in Appendix $\mathrm{C}$, it is a remarkable fact that contraction of $a(t)$ stops and reverses when $\rho_{\text {wall }}$ satisfies"

$$
\rho_{b}=n(n-1) M_{*}^{n+2} b^{n} H_{b}^{2}=\rho_{\text {wall }} .
$$

There are two generic possibilities for how this condition may come to be satisfied: The first takes the primordial $\rho_{\text {wall }}$ left over from the inflationary epoch. If there is sufficient $a(t)$ contraction, this inflationary $\rho_{\text {wall }}$ can becomes comparable to $\rho_{b}$ before $b$ reaches the stabilization point, $b_{0}$. Then a "Big Bounce" occurs, the contraction stops, and a modified expansionary phase that we discuss below begins. This expansion finally becomes the usual FRW expansion after $b$ reaches $b_{0}$. The second possibility is that a form of reheating takes place on the wall which is totally unconnected with the contraction of $a(t)$, but that again leads to $\rho_{\text {wall }} \geq \rho_{b}$. Possibilities in this class include the decay of some metastable state on the wall, or the collision of some other brane with our brane.

Consider the first, model-independent, scenario. We are used to thinking of the universe at the end of inflation as being very cold, but due to the de Sitter era Hawking radiation this is not completely so. What is the initial value of the ratio $\rho_{\text {wall }} / \rho_{b}$ so produced? At the end of inflation, our brane is not empty but inevitably contains

${ }^{* *}$ The exact analysis we have performed proves that this is the case when the dominant form of radion energy is kinetic, and, moreover, as we will show in Section 3.4, necessarily and automatically occurs if the radion stabilizes. 
a radiation bath left over from the early inflationary de Sitter phase, whose energy density at the end of the de Sitter phase has been estimated by Ford [23] (see also [24]), with the result

$$
\rho_{\text {wall }, i} \simeq 10^{-2}\left(H_{a, I}\right)^{4},
$$

depending on the exact nature (conformal or not) of the coupling between the matter and metric. Here $H_{a, I}$ is the Hubble parameter at the exit from inflation. On the other hand, the initial value of the radion energy is of order

$$
\rho_{b, i} \simeq n(n-1) M_{*}^{n+2}\left(b_{I}\right)^{n}\left(H_{b, I}\right)^{2} \simeq 6 M_{*}^{n+2}\left(b_{I}\right)^{n}\left(H_{a, I}\right)^{2},
$$

since inflation terminates when $H_{b} / H_{a}$ approaches unity. Furthermore due to the slow roll conditions, at inflation exit $H_{a}$ is roughly the same as during the inflationary phase. Given these expressions for the initial energy densities, equations (27) and (28) together with the constraint of reproducing the CMB/COBE data lead to the interesting relationship

$$
\left.\frac{\rho_{\text {wall }}}{\rho_{b}}\right|_{i} \simeq \frac{n(n-1)}{6} S^{2}\left(\left.\frac{\delta \rho}{\rho}\right|_{\mathrm{COBE}}\right)^{2}
$$

Due to the contraction of our scale factor, the wall-localized radiation can become important, the ratio $\rho_{\text {wall }} / \rho_{b}$ approaching unity. Using the fact that $\rho_{\text {wall }} \sim a^{-4}$, and the relation (70) between the evolution of $a$ and $b$, we find that it scales as

$$
\begin{array}{ll}
\frac{\rho_{\text {wall }}}{\rho_{b}} \sim \frac{b^{p}}{a^{4}} \sim b^{\left(2 n^{2}+4 n p+p^{2}-4 n\right) /(2 n+p)} & \text { for } \Delta>0 \\
\frac{\rho_{\text {wall }}}{\rho_{b}} \sim \frac{b^{-2 n+\sqrt{3 n(n+2)}}}{a^{4}} \sim b^{\sqrt{n(2+n) / 3}} & \text { for } \Delta<0 .
\end{array}
$$

Given the initial value (79) of the ratio we can compute with the aid of the scaling laws (80) how it behaves as the contraction proceeds. Putting in the experimentally observed value of $\delta \rho / \rho \sim 2 \times 10^{-5}$, and taking a reasonable value of $S \sim 0.01$, we find that in the simple Kasner $\Delta<0$ case, $\rho_{\text {wall }}=\rho_{b}$ before $b \simeq b_{0}$ for all $n=2, \ldots, 6$. On the other hand, in the case of the Kasner-like solutions with potential, $\Delta>0$, the rate of increase of the ratio $\rho_{\text {wall }} / \rho_{b}$ is always slower as a function of $b$, and in some cases (e.g., $n=3, p=0$ ) the stabilization point is reached before $\rho_{\text {wall }} \sim \rho_{b}$. We will focus on the $\Delta<0$ cases for illustration in the following.

Specifically, using the scaling laws for the evolution of the energy densities, $\rho_{\text {wall }}=\rho_{b}$ at a value of $b=b_{f}$ given by

$$
\left(\frac{b_{f}}{b_{i}}\right)=\left(\left.S \frac{\delta \rho}{\rho}\right|_{C O B E}\right)^{-2 \sqrt{n(n+2) / 3}} .
$$


We can now compute the energy density on the wall at the end of contraction:

$$
\rho_{\text {wall }, f} \simeq 7 \times 10^{2} M_{*}^{4}\left(b_{I} M_{*}\right)^{2 n} n(n-1)\left(\left.S \frac{\delta \rho}{\rho}\right|_{C O B E}\right)^{\left(2(n+1)^{2}-4 n \sqrt{n(n+2) / 3}\right)} .
$$

In terms of the temperature of radiation at the end of the contraction phase, this becomes

$$
T_{\text {wall }, f} \simeq 6 M_{*}\left(b_{I} M_{*}\right)^{n / 2}(n(n-1))^{1 / 4}\left(\left.S \frac{\delta \rho}{\rho}\right|_{C O B E}\right)^{\left((n+1)^{2} / 2-n \sqrt{n(n+2) / 3}\right)} .
$$

If we use the fact that the typical volume (measured in units of the fundamental Planck mass $M_{*}$ ) of the internal dimensions during inflation is at most $\left(b_{I} M_{*}\right)^{n} \simeq 10^{3}$, and that $S \delta \rho / \rho<2 \times 10^{-7}$, given $\mathrm{COBE}$ and $S \lesssim 0.01$, we find that the wall temperature at the end of contraction is bounded above by $1.4 \times 10^{-6}, 7 \times 10^{-7}, 4.5 \times 10^{-6}, 3 \times 10^{-4}$, and 0.22 in units of $M_{*}$, for $n=2, \ldots, 6$ respectively.

We compare this to the limits found in [3], which showed that the temperature of radiation on the brane, by stabilization, cannot exceed a certain maximal value in order to prevent overproduction of bulk gravitons by evaporation. This temperature, called the normalcy temperature $T_{*}$ in [3], was defined as the temperature above which the cooling of the wall by graviton production begins to compete with the normal adiabatic cooling by expansion. It was estimated to range between about $10 \mathrm{MeV}$ for $n=2$ to about $1 \mathrm{GeV}$ for $n=6$. For $M_{*} \sim 1 \mathrm{TeV}$, the above expression for the reheating temperature of the wall radiation at the end of contraction gives about $1.4 \mathrm{MeV}$ for $n=2$ up to about $300 \mathrm{MeV}$ for $n=5$ and $220 \mathrm{GeV}$ for $n=6$. In fact, if we take $M_{*} \simeq 30 \mathrm{TeV}$ for the $n=2$ case, as suggested in [3], we can see that the upper bound on the predicted wall reheating temperature becomes $\simeq 45 \mathrm{MeV}$. Thus we see that apart from the $n=6$ case we certainly satisfy the normalcy bounds on the reheating temperature.

However, these numbers are only the upper bounds for a number of reasons. First, in reality, the evolution is not always Kasner-like. Instead, there are periods immediately after the end of inflation when the brane scale factor is increasing, or decreasing more slowly than in the Kasner case. As a result of this, the actual blue-shift is less than the bounds used above, and this can easily lower the upper bound by an $O(1)$ factor. Second, after the end of contraction, and until the stabilization point $b=b_{0}$ is obtained, both $a(t)$ and $b(t)$ expand. Since the normalcy bounds strictly only apply after stabilization is reached, and the expansion of $a(t)$ red-shifts the temperature, the above estimates of $T_{\text {wall }}$ are actually too high. 
Having found reassurance in these numbers, we can ask if this reheating could be not only indifferent, but beneficial for late cosmology. Namely, the bounds we have derived above suggest that the reheating of the universe on the wall due to contraction may be just sufficient to warm the world enough so that nucleosynthesis can occur without hindrance. Indeed, the upper bound on the reheat temperature is always above the nucleosynthesis scale, suggesting a very interesting possibility that in this picture reheating and particle production could be purely gravitational. In conventional cosmological models based on quantum gravity at the scale $10^{19} \mathrm{GeV}$, gravitational particle production is typically insufficient for reheating, largely due to the fact that inflation occurs early, and that between it and the nucleosynthesis era the universe has expanded by many orders of magnitude, diluting the particles produced in the early de Sitter phase. In our model, in contrast, the part of the world which contains our universe contracts, increasing the energy density and number density on the wall rather than diluting them.

\subsection{Bulk graviton production}

We close this section with the consideration of production rates for bulk gravitons during the era of radion evolution. There are actually two slightly different issues here that we have not so far distinguished: i) the energy density in the (would-be) zero mode of the bulk graviton, namely the radion, and, ii) the energy density in Kaluza-Klein (KK) excitations of the graviton in the bulk.

The constraints on the energy density in KK excitations is actually more severe than that on the radion energy density, which just comes from overclosure. The reason for this is that even though the lifetime of the bulk KK modes is very long, a small fraction of them will decay back to photons on our brane, causing distortions in the diffuse gamma-ray background [3]. The diffuse gamma-ray background constraint only applies to the excited KK modes, since in general it is only the excited states that can decay to dangerous energetic SM states on our brane. (Recall that typically the mass of the radion, the would-be zero mode, is $\gtrsim 10^{-3} \mathrm{eV}$.) This constraint is more severe than the overclosure constraint which applies to both the radion zero mode and all the KK excitations together.

In any case in the next section we will consider the overclosure constraint on the total bulk energy, and see that generically there is a problem. Here we want to check that the bound on the KK excitations of the graviton is automatically satisfied. (The

analysis will show in passing that the dominant form of bulk energy will be in the 
radion motion, and not the KK excitations.)

First consider the evolution of the projected energy density (of mass dimension $\left[\rho_{K K}\right]=4$ as usual) of the bulk KK gravitons in the absence of particle production:

$$
\dot{\rho}_{K K}+3 H_{a} \rho_{K K}+H_{b} \rho_{K K}=0 .
$$

The second term on the LHS corresponds to the usual $1 / a^{3}$ dilution of massive particles (which from this 4-dimensional perspective the KK excitations of the graviton appear to be; see [3] for a full discussion). The novel feature of this equation is the third term which expresses the fact that as the internal radii increase, the $K K$ masses decrease as $1 / b$. Another way of saying this is that the KK mass is really the quantized momentum in the internal directions, and this red-shifts as $b(t)$ expands.

As recognized long-ago by Parker and Zeldovich, and clarified later by many authors [24], a time-dependent gravitational field can produce particles from the vacuum, by essentially a version of the pair-creation process that takes place in strong electric fields for charged particles. Up to order one coefficients that will not be important, the magnitude of this particle production per unit time per unit volume is given by $H^{5}$, where $H$ is the typical Hubble constant. Thus the equation for the projected KK energy density becomes

$$
\dot{\rho}_{K K}+3 H_{a} \rho_{K K}+H_{b} \rho_{K K}=H^{5} .
$$

It is easy to solve this equation. Using the substitution $\rho_{K K}=f\left(\frac{a_{i}}{a}\right)^{3}\left(\frac{b_{i}}{b}\right)$, it can be reduced to

$$
\dot{f}=H^{5}\left(\frac{a_{i}}{a}\right)^{3}\left(\frac{b_{i}}{b}\right)
$$

which, employing the Kasner era power laws, and noting that the resulting power of $t$ is less than -1 , gives upon integration a result dominated by the early stages

$$
f \sim \frac{1}{t_{i}^{4}}-\frac{1}{t^{4}} \simeq H_{i}^{4}
$$

for all power-law solutions. Converted back into $\rho_{K K}$, this says that the final energy density of the KK gravitons in the bulk which came from particle production is bounded from above by

$$
\rho_{K K, f} \lesssim H_{i}^{4}\left(\frac{a_{i}}{a_{f}}\right)^{3}\left(\frac{b_{i}}{b_{f}}\right) .
$$

\footnotetext{
${ }^{\dagger \dagger}$ More precisely, as shown in 24, in the anisotropic case considered here, it is the Hubble constant of the contracting dimensions.
} 
This differs from our estimate of the final energy density of the blue-shifted walllocalized radiation at the end of the epoch of contraction, only in that it is further suppressed by a factor of $\left(a_{f} / a_{i}\right)\left(b_{i} / b_{f}\right)$, which comes from the fact that the KK gravitons are red-shifted by the bulk expansion, but only diluted and not blue-shifted by the wall contraction. Thus we get

$$
\begin{aligned}
\frac{\rho_{K K, f}}{\rho_{\text {wall }, f}} & \leq\left(\frac{a_{f}}{a_{i}}\right)\left(\frac{b_{i}}{b_{f}}\right) \\
& \lesssim\left(10^{1-30 / n}\right)^{1+\zeta}
\end{aligned}
$$

where in the second line we have used (70), and the conservative estimates $b_{i} \sim 10 M_{*}^{-1}$ and $b_{f}=b_{0}$. Evaluating this in, for example, the case of the simple Kasner contraction with exponents given in (71) leads to $\rho_{K K} / \rho_{\text {wall }}$ varying between $3 \times 10^{-17}$ for $n=2$ to $1 \times 10^{-8}$ for $n=6$. This shows that the effective temperature of the KK gravitons is well below the diffuse gamma-ray bound, even before any dilution necessary to solve the radion moduli problem. It also demonstrates that the vast majority of the energy in the bulk is in the motion of the zero mode radion $\rho_{b}$, rather than in the bulk KK modes. This is simply because we have shown above that $\rho_{b} \simeq \rho_{\text {wall }}$ is the natural circumstance at the end of the epoch of contraction.

\subsection{The era of contraction and stabilization in the Einstein frame}

The epoch where the radius grows from its initial small size to its final value can also be simply and physically understood in the Einstein frame. (Einstein frame quantities will be denoted by an overbar in the following.) Recall that the metric in the Einstein frame is related to the one in the string frame via

$$
\bar{g}_{\mu \nu}=e^{n \bar{\beta}} g_{\mu \nu}, \quad b=e^{\bar{\beta}}
$$

(throughout this subsection we work in units with $M_{*}=1$ ). Using this map, it is straightforward to relate the quantities in the string frame to their counterparts in the Einstein frame. For instance, it is trivial to check that

$$
H_{a}=e^{\frac{n}{2} \bar{\beta}}\left(\bar{H}_{a}-\frac{n}{2} \dot{\bar{\beta}}\right)
$$

Therefore, we can see that even though in the Einstein frame $\bar{H}_{a}>0$, this can look like "contraction" in the string frame, i.e. $H_{a}<0$, provided that $\dot{\bar{\beta}}$ is large enough. On 
the other hand, as long as the radius eventually stabilizes at its final size so $\bar{\beta}=0$, we will have $H_{a}>0$. This proves that, in the string frame, there is always a "big bounce" as long as the radius eventually stabilizes.

Moving on to the dynamics, the action in the Einstein frame is

$$
S=\int d^{4} y \sqrt{-\bar{g}}\left(-\overline{\mathcal{R}}+\frac{n(n+2)}{2}(\partial \bar{\beta})^{2}-e^{-2 n \bar{\beta}} V(\bar{\beta})\right)
$$

We can parametrize the potential as

$$
V=b^{-p} W \equiv e^{-p \bar{\beta}} \bar{f}(\bar{\beta})
$$

We wish to regard $\bar{f}$ as very slowly varying and will treat it as a constant during the radion "coasting". Of course, at the final value of the radion $\beta_{*}$ we must have $\bar{f}(\beta)=\bar{f}^{\prime}(\beta)=0, \bar{f}^{\prime \prime}(\beta)>0$.

If we now work with the canonically normalized field

$$
\beta=\sqrt{n(n+2)} \bar{\beta}
$$

the action becomes

$$
S=\int d^{4} y \sqrt{-\bar{g}}\left(-\overline{\mathcal{R}}+\frac{1}{2}(\partial \beta)^{2}-e^{-x \beta} f(\beta)\right), \quad x \equiv \frac{2 n+p}{\sqrt{n(n+2)}}
$$

The equations of motion are now just the familiar ones for a FRW universe with a scalar field $\beta$ with an effective potential $V_{\text {eff }}(\beta)=e^{-x \beta} f(\beta)$. Writing the scale factor as usual $a=e^{\alpha}$, we have

$$
\begin{aligned}
& 6 \dot{\alpha}^{2}=\frac{1}{2} \dot{\beta}^{2}+f e^{-x \beta} \\
& \ddot{\beta}+3 \dot{\alpha} \dot{\beta}=x f e^{-x \beta}
\end{aligned}
$$

Suppose we ignore $f$; then $\beta$ only has kinetic energy and we trivially find the solutions

$$
\begin{array}{r}
\alpha=\frac{1}{3} \log (\mathrm{t}) \\
\beta=\frac{2}{\sqrt{3}} \log (\mathrm{t})
\end{array}
$$

Obviously, for sufficiently large $x$, the effective potential $V_{\text {eff }}$ is falling off so rapidly that ignoring $f$ should be a good approximation. We can see what the lower bound on $x$ is by substituting the above potential-free solutions into the exact equations of 
motion; the kinetic energy terms scale like $t^{-2}$, whereas the potential energy term scales like $t^{-2 x / \sqrt{3}}$. Therefore, ignoring the potential is a good approximation when

$$
x>\sqrt{3} \rightarrow x^{2}-3=-\Delta>0
$$

which agrees with the string frame result. Notice also that this case corresponds to the string frame contraction, since

$$
\operatorname{sgn}\left(H_{a}\right)=\operatorname{sgn}\left(\dot{\alpha}-\frac{n}{2 \sqrt{n(n+2)}} \dot{\beta}\right)=\operatorname{sgn}\left(\frac{1}{3}-\frac{n}{\sqrt{3 n(n+2)}}\right)<0
$$

What about $x<\sqrt{3}$ or $\Delta>0$ ? In this case, the effective potential does not fall steeply enough for the energy density to become kinetic energy dominated. What happens instead is that the radion first gets accelerated by the potential till the kinetic energy briefly dominates, whereupon it gets diluted again and the cycle repeats. Therefore, on average we expect the kinetic and potential energies to be the same. It is easy to see that this assumption is self-consistently justified by the equations. Therefore, we have

$$
\dot{\beta}=e^{-\frac{x}{2} \beta} \rightarrow \beta=\frac{2}{x} \log (\mathrm{t})
$$

Inserting this ansatz back into the equations, we find trivially

$$
\alpha=\frac{1}{x^{2}} \log (t)
$$

These solutions correspond to the new, modified Kasner solutions found in the string frame analysis. Once again, it is easy to see that these solutions correspond to contraction in the string frame:

$$
\operatorname{sgn}\left(H_{a}\right)=\operatorname{sgn}\left(\frac{1}{x^{2}}-\frac{n}{\sqrt{n(n+2) x}}\right)=\operatorname{sgn}(2-n-p)
$$

while from our solutions in the string frame $a$ contracts when $n+p-2>0$.

We can now discuss what happens as the radion nears its final minimum at $\beta=\beta_{*}$. The effective potential $V_{\text {eff }}$ can be approximated as quadratic around this final point for $\left(\beta-\beta_{*}\right) / \beta_{*} \lesssim 1$. If the radion approaches this region with kinetic energy sufficiently smaller than, or comparable to, the potential energy, then by equipartition it will be trapped in the well and will oscillate about the minimum. On the other hand, if the kinetic energy is much larger than the potential at the top of the well, $\beta$ will escape from the region close to the minimum; its subsequent fate depends on the form of $V_{e f f}$ 
at larger values of $\beta$. It can either escape to infinity, or it may turn back around at some large distance away and "slosh" back and forth with very large amplitude about $\beta_{*}$. It is therefore clear that for $x<\sqrt{3}$, where the kinetic and potential energy stay comparable throughout the coasting period, the radion will not overshoot. On the other hand, it appears that for $x>\sqrt{3}$, where the kinetic energy dominates, the radion could significantly overshoot the minimum. This statement has to be qualified, however, since all of the above analysis neglects the effect of the radiation energy density left over after inflation. Of course, in the Einstein frame,this energy does not appear to be blueshifted, because the Einstein frame scale factor never decreases. However, during the kinetic energy dominated era, the kinetic energy redshifts as $\bar{a}^{-6}$ whereas the radiation energy density only redshifts like $\bar{a}^{-4}$, so the radiation will eventually dominate. Let us consider what happens after radiation domination, ignoring the radion potential. Since $\bar{a} \sim \sqrt{t}$ during radiation domination, the radion kinetic energy $\dot{\beta}^{2} \sim \bar{a}^{-6} \sim t^{-3}$, so $\dot{\beta} \sim t^{-3 / 2}$ giving $\beta \sim t^{-1 / 2}$. In other words, ignoring the radion potential, the radiation provides enough friction to stop $\beta$. Therefore, if radiation domination happens before $\beta$ approaches the minimum $\beta_{*}$, radiation domination prevents overshoot. In all cases of interest to us this indeed happens; as demonstrated numerically in subsection 3.2, radiation domination takes place for values of the radius $b$ less than the minimum $b_{0}$.

\subsection{Stabilization and the moduli problem}

In the previous subsections we discussed a rather interesting mechanism by which the remnant Hawking radiation left over from the de Sitter era could be the source of reheating on our brane when combined with the fact that $a(t)$ goes through a period of contraction.

However as we noted above, before this mechanism can be viewed as a realistic way to reheat our brane, we must consider the radion field. At the stage where the contraction stops and the brane undergoes a Big Bounce, the contribution of the energy density of the radion field is still a significant contribution to the total (indeed $O(1)$ ). This fact leads to a radion moduli problem. The difficulty is that the radion is so light and weakly coupled that it lives typically much longer than the age of the universe. Since its coherent oscillations about the minimum redshift away only as $1 / a^{3}$, it can eventually overclose universe. In order to avoid this, the energy stored in the radion must be small, relative to the radiation energy $T_{*}^{4}$, when the universe is reheated to $T_{*}$ :

$$
\frac{\rho_{\text {rad* }}}{T_{*}^{4}}=\frac{\rho_{\text {rad* }}}{T_{*}^{3}} \times \frac{1}{T_{*}}=\frac{\rho_{\text {rad } 0}}{T_{0}^{3}} \times \frac{1}{T_{*}}<\frac{3 \times 10^{-9} \mathrm{GeV}}{T_{*}}
$$


where we have used $\rho / T^{3}=$ const. and $\rho_{\text {crit } 0} / T_{0}^{3} \sim 3 \times 10^{-9} \mathrm{GeV}$. Given that $T_{*}$ is bounded between $\sim 1 \mathrm{MeV}-1 \mathrm{GeV}$ by normalcy constraints, the energy density in the radion must somehow be diluted by $\sim 10^{-7}-10^{-9}$ in order to avoid overclosure. Fortunately this implies that, for example, only about 5 or 6 efolds of late inflation are needed so solve this problem. In any case this moduli problem is one of late (poststabilization) cosmology, rather than early pre-stabilization cosmology; we will return to address it in a future publication.

\section{Conclusions}

We have argued that early inflation when the internal dimensions are still small can successfully accomplish all that is required of inflation, including generation of suitable $\delta \rho / \rho$ without the unpleasant introduction of very light or fine-tuned wall fields. Indeed, the very fact that the internal dimensions must expand from their initial size close to the fundamental Planck length $M_{*}^{-1}$ to the stabilization value $b_{0} \simeq 10^{-17+30 / n} \mathrm{~cm}$ leads to natural inflationary scenarios involving the dynamics of the internal space. The resolution of cosmological conundrums such as the horizon, flatness and age problems, and the production of the spectrum of nearly-scale invariant Harrison-Zeldovich density perturbations with the avoidance of drastic fine tuning of the inflaton mass come as a consequence of the evolution of the internal space. Moreover it is remarkable that the era of post-inflation brane-contraction that follows this period of inflation is harmless, and automatically ends via a "Big Bounce". During the phase of $b(t)$ evolution to the stabilization point, the production of bulk gravitons by the timevarying metric remains completely suppressed, ensuring that the bulk is very cold at, and after, the stabilization of the internal dimensions. The primary remaining issue is the radion moduli problem, which is no more severe than in gauge-mediated supersymmetry breaking models. Overall, then, early universe cosmology in these models is quite interesting!

\section{Acknowledgments}

It is a pleasure to thank Gia Dvali for valuable discussions of related ideas 25] and to Andrei Linde for useful conversations. SD thanks the CERN theory group, and JMR thanks the Stanford University theory group, for their respective hospitality during

portions of this work. The work of SD and NK is supported in part by NSF grant 
PHY-9870115. The work of JMR is supported in part by an A.P. Sloan Foundation Fellowship.

\section{Appendix A: Kinematics of the radion field}

With the metric of the form (9) the Ricci scalar is

$$
-\mathcal{R}=6 \frac{\ddot{a}}{a}+6\left(\frac{\dot{a}}{a}\right)^{2}+2 n \frac{\ddot{b}}{b}+n(n-1)\left(\frac{\dot{b}}{b}\right)^{2}+6 n\left(\frac{\dot{a} \dot{b}}{a b}\right)+\frac{\kappa n(n-1)}{b^{2}}
$$

where the internal curvature term is present for $n$-spheres $(\kappa=1)$, but vanishes for tori $(\kappa=0)$, and we have ignored a similar curvature term for the large dimensions. After integrating over all spatial coordinates we obtain,

$$
S=\int d t\left(\mathcal{L}_{\mathrm{KE}}(\dot{a}, \dot{b})-a^{3} V(b)\right)
$$

and further integrating the $\ddot{a}$ and $\ddot{b}$ terms by parts, the kinetic part of the action for the radii, $a$ and $b$, becomes

$$
S=-M_{*}^{2+n} \int d t a^{3} b^{n}\left(6\left(\frac{\dot{a}}{a}\right)^{2}+n(n-1)\left(\frac{\dot{b}}{b}\right)^{2}+6 n\left(\frac{\dot{a} \dot{b}}{a b}\right)\right) .
$$

Note the overall negative sign of these kinetic terms, but also the mixing between $\dot{a}$ and $\dot{b}$.

There is clearly an extremum of the action with $\dot{a}=\dot{b}=0$, when the condition $\left.\partial_{a}\left(a^{3} V_{\text {tot }}(b)\right)\right|_{a=a_{0}, b=b_{0}}=0$, and similar with $\partial_{a} \rightarrow \partial_{b}$ are met. These imply (for $a_{0} \neq 0$ )

$$
\begin{aligned}
& V_{\text {tot }}\left(b_{0}\right)=0, \quad \text { and } \\
& V_{\text {tot }}^{\prime}\left(b_{0}\right)=0 .
\end{aligned}
$$

This is as one would have naively expected. However, because of the negative sign for the kinetic term for the radial degrees of freedom, the stability analysis for such static solutions has to be treated with care. The analysis starts by expanding the action, Eq. (106), in small fluctuations around the extremum: $a(t)=a_{0}+\delta a(t)$, and $b(t)=b_{0}+\delta b(t)$. Then to quadratic order, and defining $\Delta \equiv \delta a / a_{0}$ and $\delta \equiv \delta b / b_{0}$, the expansion gives the coupled equations of motion

$$
\left(\begin{array}{cc}
6 & 3 n \\
3 n & n(n-1)
\end{array}\right)\left(\begin{array}{c}
\ddot{\Delta} \\
\ddot{\delta}
\end{array}\right)=\left(\begin{array}{cc}
0 & 0 \\
0 & \omega^{2}
\end{array}\right)\left(\begin{array}{c}
\Delta \\
\delta
\end{array}\right)
$$


where

$$
\omega^{2}=\frac{1}{2} \frac{\left(b_{0}\right)^{2} V_{\mathrm{tot}}^{\prime \prime}\left(b_{0}\right)}{M_{*}^{2+n}\left(b_{0}\right)^{n}}=\frac{1}{2} \frac{\left(b_{0}\right)^{2} V_{\mathrm{tot}}^{\prime \prime}\left(b_{0}\right)}{M_{\mathrm{pl}}^{2}} .
$$

is the radion mass around the stabilization point. Searching for oscillating solutions, $(\Delta, \delta)=\exp (i \Omega t)\left(\Delta_{0}, \delta_{0}\right)$ of the stability equations leads to an eigenvalue problem for the frequency $\Omega$. Specifically, $\Omega^{2}$ has the eigenvalues $\Omega^{2}=0$, and

$$
\Omega^{2}=\frac{2}{n(2+n)} \omega^{2}
$$

The zero eigenvalue just corresponds to the fact that $a_{0}$ is a flat direction since, by assumption, there is no potential for $a$. The crucial expression is Eq. (110), which gives the condition for stability of the static solution. In the end, stability just requires that the radion (mass) ${ }^{2}$ be positive as one would expect, and that we can think in terms of a total potential $V(b)$ that one can minimize to find the stable static solutions for the size of the internal dimensions.

The equations of motion for $a(t)$ and $b(t)$ derived from the action Eqs. (105) and (106) are, after some algebra, those given in the text, namely (10), (11) and the constraint (12) which comes about from the well-known property that the total energy in GR is zero. (This constraint can be derived by carefully working in terms of the lapse and shift functions of the canonical formalism.) If matter on our wall is also included then they become those given in $(57)$ :

$$
\begin{aligned}
& 6 H_{a}^{2}+n(n-1) H_{b}^{2}+6 n H_{a} H_{b}=\frac{V+\rho}{M_{*}^{n+2} b^{n}} \\
& \frac{\ddot{b}}{b}+(n-1) H_{b}^{2}+3 H_{b} H_{a}=\frac{1}{M_{*}^{n+2} b^{n}}\left(\frac{2 V}{n+2}-\frac{b}{n(n+2)} \frac{\partial V}{\partial b}+\frac{\rho-3 p}{2(n+2)}\right) \\
& \frac{\ddot{a}}{a}+2 H_{a}^{2}+n H_{b} H_{a}=\frac{1}{M_{*}^{n+2} b^{n}}\left(\frac{b}{2(n+2)} \frac{\partial V}{\partial b}-\frac{n-2}{2(n+2)} V+\frac{\rho+(n-1) p}{2(n+2)}\right) \\
& \dot{\rho}+3 H_{a}(p+\rho)=0 .
\end{aligned}
$$

Note that in these equations the effect of wall-localized matter is just some extra contribution to the $a(t)$ and $b(t)$ scale-factor evolution. Of course the energy density on the brane in general distorts the geometry of the internal space (as does that present on other branes that may exist in the bulk), but as far as the overall properties and evolution of the zero mode size modulus $b(t)$ of the internal space is concerned, it is correct to treat the energy density on the wall as just averaged over the whole space, as done on the RHS of these equations. 
It is useful to summarize some basic properties of the evolution equations. Since the total potential energy $U(a, b, \psi)$ is given in terms of the effective 4-dimensional potential energy density $V(b, \psi)$ by $U=a^{3} V(b, \psi)$, a uniform bulk cosmological constant is represented by $V=b^{n} \Lambda$. Substituting this form into the RHS of these equations we see that a positive bulk cosmological constant term has the effect of wanting to increase both $\ddot{a}$ and $\ddot{b}$ if the evolution of $a, b$ are studied close to zero. This is not inconsistent with the stability criteria derived above since this was explicitly the stability analysis around a stationary point of the equations with non-zero values of both $a$ and $b$. Indeed, the stability analysis can be derived directly from the equations of motion, as it must. Concretely, if we expand around a point $\left(a_{*}, b_{*}\right)$ with $V\left(b_{*}\right)=V^{\prime}\left(b_{*}\right)=0$, then linearizing the equations of motion gives

$$
\left(\begin{array}{c}
\delta \ddot{a} \\
\delta \ddot{b}
\end{array}\right)=\left(\begin{array}{cc}
0 & \frac{a_{*} b_{*}^{1-n}}{2(n+2) M_{*}^{n+2}} V^{\prime \prime} \\
0 & -\frac{b_{*}^{2-n}}{n(n+2) M_{*}^{n+2}} V^{\prime \prime}
\end{array}\right)\left(\begin{array}{c}
\delta a \\
\delta b
\end{array}\right),
$$

which exactly reproduces the previous stability analysis, in particular the requirement $V^{\prime \prime}\left(b_{*}\right)>0$ for $a_{*}, b_{*}>0$.

Another basic property that is useful to keep in mind is the effect of some small amount of wall-localized matter on the position and mass of the radion. Linearizing the equations (57) with such matter in the shift $\delta_{b}=\delta b / b_{0}$ around the stabilization point we find

$$
\ddot{\delta}_{b}+3 \dot{\delta}_{b} H_{a}=-\frac{1}{(n+2) b_{0}^{n} M_{*}^{n+2}}\left(\frac{b_{0}^{2} V^{\prime \prime}}{n}+\frac{n(\rho-3 p)}{2}\right) \delta_{b}+\frac{(\rho-3 p)}{2(n+2) b_{0}^{n} M_{*}^{n+2}} .
$$

This shows that wall-localized matter has two effects o the radion. First, the $\delta_{b^{-}}$ independent term on the RHS shifts the radion from the stabilization point by a small amount, and second the $(\rho, p)$-dependent in the parentheses on the RHS shifts the eigenfrequency of oscillations of the radion (or equivalently the radion mass). Both these effects are to be expected, and are harmless for wall-localized matter densities and pressures $\rho, p \ll M_{*}^{4}$.

\section{Appendix B: Kasner-like solutions with potential}

The solutions of (68) take different form, controlled by whether $C_{2}$ vanishes or not and the sign of $\Delta$. Let us first consider the case $\Delta>0$. Then, when $C_{2}=0$, the equation (68) simplifies to $X^{\prime}= \pm \sqrt{2 \frac{\Delta}{n(n+2)} \omega} \exp (X / 2)$, which can be integrated to

give $\exp (X)=\frac{2 n(n+2)}{\Delta \omega} \frac{1}{\tau^{2}}$. Here we have removed an additional integration constant 
by a time translation. It is straightforward now to use this and (63) to determine the solutions for $\alpha$ and $\beta$. Since $\alpha=\frac{(2 n+p)(n-p) C_{1}}{4 \Delta}-\frac{n(n+p-2)}{2 \Delta} X$ and $\beta=\frac{2 n+p}{\Delta} X-\frac{3(2 n+p) C_{1}}{2 \Delta}$, we find

$$
\begin{aligned}
e^{\alpha} & =\exp \left(\frac{(2 n+p)(n-p) C_{1}}{4 \Delta}\right)\left(\frac{\Delta \omega}{2 n(n+2)}\right)^{\frac{n(n+p-2)}{2 \Delta}} \tau^{\frac{n(n+p-2)}{\Delta}} \\
e^{\beta} & =\exp \left(-\frac{3(2 n+p) C_{1}}{2 \Delta}\right)\left(\frac{2 n(n+2)}{\Delta \omega}\right)^{\frac{2 n+p}{\Delta}} \frac{1}{\tau^{\frac{4 n+2 p}{\Delta}}} .
\end{aligned}
$$

We can now use (61) to transform (114) back to using the comoving time on the brane. Integrating (61), we find

$$
\tau \sim t^{-\frac{\Delta}{(n+p)(2 n+p)}}
$$

Note the important feature of this map, that it maps the comoving future $t \rightarrow \infty$ to the origin of "time" $\tau$ and vice-versa. Thus to discover the correct long-time asymptotics of the solutions we must extract the small- $\tau$ behavior. This is true for the general case $C_{2} \neq 0$ as well.

In any case, for $C_{2}=0$, and after appropriate rescalings, we find the behavior of the scale factors $a(t)$ and $b(t)$ to be

$$
\begin{aligned}
a & =a_{i}\left(\frac{t}{t_{i}}\right)^{-\frac{n(n+p-2)}{(n+p)(2 n+p)}} \\
b & =b_{i}\left(\frac{t}{t_{i}}\right)^{\frac{2}{n+p}}
\end{aligned}
$$

These solutions describe the cases where the brane length scales shrink while the radion continues to grow. This means that even if the brane scale factor $a(t)$ continues to expand by inertia after the end of inflation, soon after $a(t)$ starts to contract. Moreover, the behavior (116) is an asymptotic attractor for the generic cases with $C_{2}<0$ and $\Delta>0$, as we will now discuss.

Let us now consider the case when $C_{2}<0$. Again, note from (63) that this corresponds to the situation where initially both $H_{a}$ and $H_{b}$ are positive, when inflation has just ended. The equation (68) can be solved in this case by the substitution

$$
e^{-X}=\frac{8 \Delta \omega}{3(2 n+p)^{2} C_{2}^{2}} \sinh ^{2}(\vartheta)
$$

which reduces to the differential equation $\vartheta^{\prime}=\left(\frac{3(2 n+p)^{2}}{16 n(n+2)}\right)^{1 / 2} C_{2}$, whose solution is $\vartheta=$ $\left(\frac{3(2 n+p)^{2}}{16 n(n+2)}\right)^{1 / 2} C_{2} \tau$, after the appropriate choice of the coordinate origin of $\tau$. Therefore, 
the solution of 68 is

$$
e^{-X}=\frac{8 \Delta \omega}{3(2 n+p)^{2} C_{2}^{2}} \sinh ^{2}\left(\sqrt{\frac{3(2 n+p)^{2}}{16 n(n+2)}} C_{2} \tau\right)
$$

Note that the RHS is a positive semidefinite function, guaranteeing the reality of the metric, as required. Also note that the solution (118) is defined in open interval $\tau \in\left(0^{+}, \infty\right)$ and $\left(-\infty, 0^{-}\right)$, by time-reversal. Using this equation and (63), we can extract the solutions for $\alpha$ and $\beta$ :

$$
\begin{aligned}
e^{\alpha} & =\left(\frac{8 \Delta \omega}{3(2 n+p)^{2} C_{2}^{2}}\right)^{\frac{n(n+p-2)}{\Delta}} e^{\frac{(2 n+p)(n-p)}{4 \Delta}\left(C_{1}+C_{2} \tau\right)} \sinh ^{\frac{n(n+p-2)}{\Delta}}\left(\sqrt{\frac{3(2 n+p)^{2}}{16 n(n+2)}}\left|C_{2} \tau\right|\right) \\
e^{\beta} & =\left(\frac{3(2 n+p)^{2} C_{2}^{2}}{8 \Delta \omega}\right)^{\frac{2 n+p}{\Delta}} \frac{\exp \left(-\frac{3(2 n+p)}{2 \Delta}\left(C_{1}+C_{2} \tau\right)\right)}{\sinh ^{2 \frac{2 n+p}{\Delta}}\left(\sqrt{\frac{3(2 n+p)^{2}}{16 n(n+2)}}\left|C_{2} \tau\right|\right)}
\end{aligned}
$$

where the argument of the hyperbolic sine is taken to be positive, to make sure that the solutions remain real. In general, it is not possible to explicitly determine the integrated form of the gauge transformation (61). However, the asymptotic limits $\tau \rightarrow 0, \infty$ are easy to deduce, and are sufficient for our purpose here.

Taking the $\tau \rightarrow 0$ limits of (119) we find that (119) reduce precisely to (114), implying that the integrated form of (61) approaches (115). Hence in the case $C_{2}<0$ the scale factors again approach (69) as $t \rightarrow \infty$, which are therefore the appropriate future attractors in all cases $\Delta>0, C_{2} \leq 0$.

It is also amusing to consider the short $t$-time behavior of the exact solutions in this case. We find that as $\tau \rightarrow \infty$,

$$
\begin{aligned}
& e^{\alpha} \sim \exp \left(\frac{(2 n+p) C_{2} \tau}{4 \Delta}\left(n-p-n(n+p-2) \sqrt{\frac{3}{n(n+2)}}\right)\right) \\
& e^{\beta} \sim \exp \left(-\frac{(2 n+p) C_{2} \tau}{2 \Delta}\left(3-(2 n+p) \sqrt{\left.\frac{3}{n(n+2)}\right)}\right)\right.
\end{aligned}
$$

Using this, we can integrate (61) and find

$$
t \sim \exp \left(\frac{(2 n+p) C_{2} \tau}{4 \Delta}\left(n(n-p+6) \sqrt{\frac{3}{n(n+2)}}-3(n+p)\right)\right)
$$

and upon substituting this back into (120), we can show that the solutions approach from below the asymptotic expressions

$$
e^{\alpha} \rightarrow\left(\frac{t}{t_{i}}\right)^{\frac{3+\sqrt{3 n(n+2}}{3(n+3)}}
$$




$$
e^{\beta} \rightarrow\left(\frac{t}{t_{i}}\right)^{\frac{n-\sqrt{3 n(n+2)}}{n(n+3)}}
$$

The dependence on the parameter $p$ apparent in (120) has completely disappeared. This can be most easily seen by taking the derivatives with respect to $p$ of the powers which are obtained by substituting (121) into (120), noting that they are identically zero, and then setting $p=0$ to obtain (122). Also note that the resulting powers are identical to those found in the simple Kasner case (54), with lower sign taken. Thus in the very early time limit, this suggests that the universe was expanding while the radion was decreasing. However, this phase is cut out by a stage of inflation, and in fact only a very short portion is retained where both $a$ and $b$ are growing for short time, to match onto the post-inflationary era.

Now we consider the last case, $\Delta<0$, which we argued in the text should be described by the traditional potential-free Kasner solutions in the long-time limit. We can see this explicitly by examining the exact solutions once again. First note that, in this case, $C_{2}$ must be nonzero, as can be immediately seen from (68). The exact solutions can be found by the substitution

$$
e^{-X}=\frac{8|\Delta| \omega}{3(2 n+p)^{2} C_{2}^{2}} \cosh ^{2}(\vartheta)
$$

which is analogous to (117). The equation for $\vartheta$ is the same as before, and hence the solution is

$$
e^{-X}=\frac{8|\Delta| \omega}{3(2 n+p)^{2} C_{2}^{2}} \cosh ^{2}\left(\sqrt{\frac{3(2 n+p)^{2}}{16 n(n+2)}} C_{2} \tau\right)
$$

The similarity of this solution to (118) allows us to extract the expressions for $\alpha$ and $\beta$ quite easily. We find

$$
\begin{aligned}
e^{\alpha} & =\left(\frac{8|\Delta| \omega}{3(2 n+p)^{2} C_{2}^{2}}\right)^{\frac{n(n+p-2)}{|\Delta|}} e^{\frac{(2 n+p)(n-p)}{4|\Delta|}\left(C_{1}+C_{2} \tau\right)} \cosh ^{\frac{n(n+p-2)}{|\Delta|}}\left(\sqrt{\frac{3(2 n+p)^{2}}{16 n(n+2)}}\left|C_{2} \tau\right|\right) \\
e^{\beta} & =\left(\frac{3(2 n+p)^{2} C_{2}^{2}}{8|\Delta| \omega}\right)^{\frac{2 n+p}{|\Delta|}} \frac{\exp \left(-\frac{3(2 n+p)}{2|\Delta|}\left(C_{1}+C_{2} \tau\right)\right)}{\cosh ^{2 \frac{2 n+p}{\Delta \Delta}}\left(\sqrt{\frac{3(2 n+p)^{2}}{16 n(n+2)}}\left|C_{2} \tau\right|\right)}
\end{aligned}
$$

Note however the important difference between these solutions and (119). In this case, the solutions are defined on the whole interval $(-\infty, \infty)$. By considering the limits $\tau \rightarrow \pm \infty$, we can see that the solutions (125) in fact interpolate between the simple Kasner solutions (52), where for $C_{2}<0$ it starts out with powers given by (54) with lower sign and transmutes due to the intermediate potential-dominated region into (52) 
with powers (54) with upper sign taken. In reality, the far past of these solutions is cut out by the inflationary era, and again only a very short portion where both $a$ and $b$ are growing is retained, which is matched onto the post-inflationary era. All of these solutions therefore flow towards simple Kasner solutions with the powers with upper sign in (54), which are the appropriate future attractors.

\section{Appendix C: Exact Solutions for the Big Bounce}

To see that bounce behavior does indeed occur in the presence of radiation on the wall we go back to our equations of motion but now set the potential to zero and just keep the wall-localized radiation terms on the RHS:

$$
\begin{aligned}
& 6 H_{a}^{2}+n(n-1) H_{b}^{2}+6 n H_{a} H_{b}=\frac{\rho}{M_{*}^{n+2} b^{n}} \\
& \frac{\ddot{b}}{b}+(n-1) H_{b}^{2}+3 H_{b} H_{a}=\frac{\rho-3 p}{2(n+2) M_{*}^{n+2} b^{n}} \\
& \frac{\ddot{a}}{a}+2 H_{a}^{2}+n H_{b} H_{a}=\frac{1}{M_{*}^{n+2} b^{n}} \frac{\rho+(n-1) p}{2(n+2)} \\
& \dot{\rho}+3 H_{a}(p+\rho)=0 .
\end{aligned}
$$

These can again be solved exactly, and provide a good approximation for the exit from the Kasner-like phase of $a(t)$-contraction.

Set $p=\rho / 3$ for radiation. The solution for the radiation energy density is as usual

$$
\rho=\frac{B M_{*}^{n+2}}{a^{4}}
$$

(with some normalization $B$ ) and define variables $\alpha$ and $\beta$ by

$$
\begin{aligned}
a & =a_{0} e^{\alpha} \\
b & =b_{0} e^{\beta}
\end{aligned}
$$

where $a_{0}$ and $b_{0}$ are constants, determined by the values of $a$ and $b$ at the end epoch when the radion potential becomes small compared to the wall-localized radiation. Using this, the equations of motion can be rewritten as

$$
\begin{aligned}
& 6 \dot{\alpha}^{2}+n(n-1) \dot{\beta}^{2}+6 n \dot{\alpha} \dot{\beta}=A e^{-n \beta-4 \alpha} \\
& \ddot{\beta}+\dot{\beta}(3 \dot{\alpha}+n \dot{\beta})=0 \\
& \ddot{\alpha}+\dot{\alpha}(3 \dot{\alpha}+n \dot{\beta})=\frac{A}{6} e^{-4 \alpha-n \beta}
\end{aligned}
$$


where $A=B /\left(a_{0}^{4} b_{0}^{n}\right)$. If we change the time variable, defining the new time $\tau$ as in eq. (61):

$$
d \tau=-e^{-3 \alpha-n \beta} d t
$$

where again the specific form of $\tau=\tau(t)$ can be found later when solutions are determined, we can rewrite the equations of motion as

$$
\begin{aligned}
& 6 \alpha^{2}+n(n-1) \beta^{2}+6 n \alpha^{\prime} \beta^{\prime}=A e^{2 \alpha+n \beta} \\
& \beta^{\prime \prime}=0 \\
& \alpha^{\prime \prime}=\frac{A}{6} e^{2 \alpha+n \beta}
\end{aligned}
$$

where the primes denote derivatives with respect to $\tau$. The $\beta$ equation immediately gives

$$
\beta=C_{1}+C_{2} \tau
$$

Hence note that if $H_{b}>0$ initially then the constant $C_{2}<0$. Also note that given our conventions we again have future $t$ infinity map to $\tau=0$.

For the purpose of examining the solutions to these equations we distinguish two cases:

(i) $C_{2}=0$; like before, this solution turns out to be a future attractor of all solutions!

This case has a very simple analysis: $C_{2}=0$ implies $\beta^{\prime}=0$ but also $\dot{\beta}=0$. Hence we can forget about the $\tau$ coordinates and immediately work in our original brane-time $t$. So $\dot{b}=0$ means $b=b_{0}=$ const and so the constraint equation gives

$$
6 H_{a}^{2}=\frac{A}{b_{0}^{n} a^{4}}
$$

which is immediately solved to give us the radiation dominated universe, $a \sim \sqrt{t}$. This is precisely as we would expect from an analysis in the Einstein frame.

(ii) $C_{2} \neq 0$; this case corresponds to more generic solutions. In particular we will see that we can have initial conditions $H_{b}>0, H_{a}<0$, which evolve to $H_{b}=0$, $H_{a}>0$, in other words a "bounce" solution.

To solve the equations in this case, first define

$$
X=2 \alpha+n \beta
$$


in terms of which, the second order differential equations produce

$$
X^{\prime \prime}=\frac{A}{3} e^{X}
$$

This is again the Liouville equation, with the first integral (conservation of energy)

$$
X^{\prime 2}=\frac{2 A}{3} e^{X}+Z_{0}
$$

where $Z_{0}$ is another integration constant. If we eliminate $e^{X}$ from the constraint equation by using the last equation, and then simplify the result by using the second equation, we find that the constraint reduces to

$$
Z_{0}=\frac{n(n+2)}{3} C_{2}^{2} \geq 0
$$

Thus the constant $C_{2}$ controls the dynamics, and the whole system has collapsed down to two simple equations, one of which is already solved:

$$
\begin{aligned}
& \beta=C_{1}+C_{2} \tau \\
& X^{\prime 2}=\frac{2 A}{3} e^{X}+\frac{n(n+2)}{3} C_{2}^{2} .
\end{aligned}
$$

Note that the solution to the $X$ equation is,

$$
e^{X}=\frac{n(n+2) C_{2}^{2}}{2 A} \frac{1}{\sinh ^{2}\left(\sqrt{\frac{n(n+2)}{12}} C_{2} \tau\right)}
$$

as may be checked by substitution. Using the formula for $X=2 \alpha+n \beta$ and $\beta=$ $C_{1}+C_{2} \tau$, we find that

$$
e^{\alpha}=+\left(\frac{n(n+2) C_{2}^{2}}{2 A}\right)^{1 / 2} \frac{e^{-n C_{1} / 2+n\left|C_{2}\right| \tau / 2}}{\sinh \left(\sqrt{\frac{n(n+2)}{12}}\left|C_{2}\right| \tau\right)},
$$

where since $C_{2}<0$ in the cases of interest we have taken the appropriate branch of the square-root such that $a=a_{0} e^{\alpha}$ is positive as it must be.

This expression is already sufficient to show that we get a bounce behavior for $a(t)$. Recognizing that $t=0$ corresponds to $\tau \rightarrow \infty$ while $t \rightarrow \infty$ corresponds to $\tau \rightarrow 0$, simply plotting $e^{\alpha}$ as given by (140) shows that $a(t)$ goes through a bounce.

It is instructive to see this in detail. First, consider the limit $\tau \rightarrow 0$. Clearly, $\beta=C_{1}+C_{2} \tau \rightarrow C_{1}=$ const. Also,

$$
e^{\alpha} \rightarrow+\left(\frac{6 e^{-n C_{1}}}{A}\right)^{1 / 2} \frac{1}{\tau}
$$


Thus $\exp (3 \alpha+n \beta) \rightarrow \tau^{-3}$, and so we have

$$
d t \sim-\frac{d \tau}{\tau^{3}}
$$

or, $t \sim \tau^{-2}$ which gives us $\tau \sim 1 / \sqrt{t}$, and $t \rightarrow \infty$ maps to $\tau \rightarrow 0^{+}$as claimed. Back in the formula for $a$, this gives

$$
a \sim \exp (\alpha) \sim \frac{1}{\tau} \sim \sqrt{t}
$$

and this is precisely a radiation-dominated universe at late times! So indeed, the solutions with $C_{2}=0$ are late time attractors. Some further analysis shows that in this limit, (and in the approximation of ignoring the stabilizing potential) $b$ will tend to a constant logarithmically. The end result of the analysis is that for large $t$ these solutions show that $a$ is expanding asymptotically as $\sqrt{t}$.

Let's now look at the other limit, $\tau \rightarrow \infty$. Also, recall that $C_{2}$ must be less than zero: $C_{2}<0$. We have

$$
b \sim \exp (\beta) \sim \exp \left(-\left|C_{2}\right| \tau\right)
$$

and

$$
a \sim \exp (\alpha) \sim \exp \left(\frac{\left|C_{2}\right| \tau}{2}(n-\sqrt{n(n+2) / 3})\right) .
$$

These give the relationship between $\tau$ and $t$

$$
d t \sim-\exp \left(\frac{\left|C_{2}\right| \tau}{2}(n-\sqrt{3 n(n+2)})\right) d \tau
$$

Now note that $(n-\sqrt{3 n(n+2)})<0$ for all $2 \leq n \leq 6$, and therefore as $\tau \rightarrow+\infty$ we have $t \rightarrow 0^{+}$as

$$
t \sim \exp \left(\frac{-\left|C_{2}\right| \tau}{2}(\sqrt{3 n(n+2)}-n)\right)
$$

as claimed. Finally, in the expression (145) for $a(t)$, note that $(n-\sqrt{n(n+2) / 3})>0$, so as $\tau \rightarrow \infty$, the scale factor $a(t)$ is again going to infinity. Re-expressed in terms of $t$ we see that as $t$ initially increases $a(t)$ is initially decreasing.

If one carefully considers this limit the power law behavior of $a$ and $b$ in terms of the time $t$ precisely corresponds to the late-time Kasner solutions we found in Appendix B. Most importantly, we see that as $\tau$ decreases from $\infty$ towards $0^{+}$, the radion $b$ is initially increasing and $a$ is initially decreasing, the initial conditions that we require. But as we have seen from the analysis in the $\tau \rightarrow 0$ limit, this goes over to $b$ growing 
logarithmically and $a$ increasing as $1 / \tau$. This implies there must indeed have been a Big Bounce in between!

Finally, the bounce occurs when $\dot{\alpha}=0$ or equivalently $H_{a}=0$. This happens when

$$
n(n-1) H_{b}^{2}=\frac{\rho_{w a l l}}{M_{*}^{n+2} b^{n}} .
$$

Hence, when the bounce occurs the radion kinetic energy is comparable to the wall energy density. By continuity, it should be clear that the generic qualitative features of these properties would remain true even in the presence of stabilizing potentials. The main conclusion of this analysis is that the Big Bounce is the future asymptotic attractor of all postinflationary solutions with wall radiation, and hence the exit from the contraction on the wall will occur naturally.

\section{References}

[1] N. Arkani-Hamed, S. Dimopoulos and G. Dvali, Phys. Lett. B429, 263 (1998).

[2] I. Antoniadis, N. Arkani-Hamed, S. Dimopoulos and G. Dvali, Phys. Lett. B436, 257 (1998).

[3] N. Arkani-Hamed, S. Dimopoulos and G. Dvali, hep-ph/9807344.

[4] N. Arkani-Hamed, S. Dimopoulos and J. March-Russell, hep-th/9809124.

[5] J. Polchinski, Phys. Rev. Lett. 75 (1995) 4724;

Also see for example: J. Polchinski, TASI lectures on D-branes, hep-th/9611050;

C. Bachas, Lectures on D-branes, hep-th/9806199.

[6] K. Dienes, E. Dudas and T. Gherghetta, Phys. Lett. B436, 55 (1998); Nucl. Phys. B537 (1999) 47.

[7] I. Antoniadis, Phys. Lett. B246 (1990) 377;

I. Antoniadis and K. Benakli, Phys. Lett. B326 (1994) 69;

I. Antoniadis, K. Benakli and M. Quiros, Phys. Lett. B331 (1994) 313.

[8] P. Horava and E. Witten, Nucl. Phys. B460 (1996) 506;

E. Witten Nucl. Phys. B471 (1996) 135;

J.D. Lykken, Phys. Rev. D54 (1996) 3693;

E. Caceres, V.S. Kaplunovsky and I.M. Mandelberg, Nucl. Phys. B493 (1997) 73. 
[9] G. Shiu and S.H. Tye, Phys. Rev. D58, 106007 (1998).

[10] C. Bachas, hep-ph/9807415;

I. Antoniadis and C. Bachas, hep-th/9812093.

[11] R. Sundrum, hep-ph/9805471.

[12] R. Sundrum, hep-ph/9807348.

[13] P. Argyres, S. Dimopoulos and J. March-Russell, hep-th/9808138;

Z. Kakushadze and S.-H. Tye, hep-th/9809147;

K. Dienes, et al., hep-ph/9809406;

K. Benakli, hep-ph/9809582;

L. Randall and R. Sundrum, hep-th/9810155;

G.F. Giudice, R. Rattazzi and J.D. Wells, hep-ph/9811291;

S. Nussinov and R. Shrock, hep-ph/9811323;

E.A. Mirabelli, M. Perelstein and M.E. Peskin, hep-ph/9811337;

T. Han, J.D. Lykken and R. Zhang, hep-ph/9811350;

J.L. Hewett, hep-ph/9811356;

Z. Berezhiani and G. Dvali, hep-ph/9811378;

K.R. Dienes, E. Dudas and T. Gherghetta, hep-ph/9811428;

N. Arkani-Hamed, et al., hep-ph/9811448;

Z. Kakushadze, hep-th/9812163.

[14] K. Benakli and S. Davidson, hep-ph/9810280.

[15] D. Lyth, hep-ph/9810320.

[16] N. Kaloper and A. Linde, hep-th/9811141.

[17] G. Dvali and S.H. Tye, hep-ph/9812483.

[18] A. Mazumdar, hep-ph/9902381.

[19] A. Linde, Phys. Lett. B129, 177 (1983).

[20] A. D. Linde, Particle Physics and Inflationary Cosmology (Harwood, Chur, Switzerland, 1990).

[21] See for example:

C. Mathiazhagan and V.B. Johri, Class. Quant. Grav. 1, L29 (1984); 
F. Lucchin and S. Matarrese, Phys. Rev. D32 (1985) 1316;

A.B. Burd and J.D. Barrow, Nucl. Phys. B308 (1988) 929.

[22] S. Kalara, N. Kaloper and K.A. Olive, Nucl. Phys. B341 (1990) 252.

[23] L. Ford, Phys. Rev. D35 (1987) 2955.

[24] See: L. Parker, in Asymptotic Structure of Space-Time, Cincinnati 1976, eds. F. Esposito and L. Witten, (Plenum, New York); Y.B. Zeldovich, JETP Lett. 12 (1970) 307.

[25] G. Dvali, in preparation. 